\title{
Mesodermal iPSC-derived progenitor cells functionally regenerate cardiac and skeletal muscle
}

\author{
Mattia Quattrocelli, ${ }^{1}$ Melissa Swinnen, ${ }^{2}$ Giorgia Giacomazzi, ${ }^{1}$ Jordi Camps, ${ }^{1}$ Ines Barthélemy, ${ }^{3}$ Gabriele Ceccarelli, ${ }^{4}$ Ellen Caluwé, ${ }^{2}$ \\ Hanne Grosemans, ${ }^{1}$ Lieven Thorrez, ${ }^{1}$ Cloria Pelizzo, ${ }^{5}$ Manja Muijtjens, ${ }^{6}$ Catherine M. Verfaillie, ${ }^{6}$ Stephane Blot, ${ }^{3}$ Stefan Janssens, ${ }^{2}$ \\ and Maurilio Sampaolesi ${ }^{1,4}$ \\ ${ }^{1}$ Translational Cardiomyology Laboratory, Embryo and Stem Cell Biology, Department of Development and Regeneration, and ²Cardiology, Department of Cardiovascular Sciences, KU Leuven, Leuven, \\ Belgium. ${ }^{3}$ Unité de Neurologie, Ecole Nationale Vétérinaire d’Alfort, Université Paris Est, Paris, France. ${ }^{4}$ Division of Human Anatomy, University of Pavia, Pavia, Italy. ${ }^{5}$ Department of Maternal and Children's \\ Health, Pediatric Surgery Unit, Fondazione IRCCS Policlinico San Matteo, Pavia, Italy. ${ }^{6}$ Stem Cell Institute Leuven, Embryo and Stem Cell Biology, Department of Development and Regeneration, KU Leuven, \\ Leuven, Belgium.
}

\begin{abstract}
Conditions such as muscular dystrophies (MDs) that affect both cardiac and skeletal muscles would benefit from therapeutic strategies that enable regeneration of both of these striated muscle types. Protocols have been developed to promote induced pluripotent stem cells (iPSCs) to differentiate toward cardiac or skeletal muscle; however, there are currently no strategies to simultaneously target both muscle types. Tissues exhibit specific epigenetic alterations; therefore, source-related lineage biases have the potential to improve iPSC-driven multilineage differentiation. Here, we determined that differential myogenic propensity influences the commitment of isogenic iPSCs and a specifically isolated pool of mesodermal iPSC-derived progenitors (MiPs) toward the striated muscle lineages. Differential myogenic propensity did not influence pluripotency, but did selectively enhance chimerism of MiP-derived tissue in both fetal and adult skeletal muscle. When injected into dystrophic mice, MiPs engrafted and repaired both skeletal and cardiac muscle, reducing functional defects. Similarly, engraftment into dystrophic mice of canine MiPs from dystrophic dogs that had undergone TALEN-mediated correction of the MD-associated mutation also resulted in functional striatal muscle regeneration. Moreover, human MiPs exhibited the same capacity for the dual differentiation observed in murine and canine MiPs. The findings of this study suggest that MiPs should be further explored for combined therapy of cardiac and skeletal muscles.
\end{abstract}

\section{Introduction}

Induced pluripotent stem cells (iPSCs) represent a promising contribution to regenerative medicine (1). Despite the regulatory hurdles and safety issues involved, reprogramming patients' cells into iPSCs for autologous cell therapy holds potential for degenerative disorders such as muscular dystrophies (MDs) (2). Albeit highly heterogeneous in their genetic etiology, many forms of MDs cause not only progressive deterioration of skeletal muscles, but also chronic degeneration of cardiac tissue $(3-5)$. Therefore, MD treatment would ideally encompass the regeneration of both striated muscle types. Several protocols have been described for the differentiation of iPSCs toward cardiac or skeletal muscle progenitors $(6,7)$, yet a single strategy to target both muscle types in vivo remains elusive.

Several reports in recent years have shown that some tissue-specific epigenetic biases are maintained in reprogrammed cells, thus leading to the so-called epigenetic memory in iPSCs (8, 9). If sufficiently durable, the epigenetic bias results in a skewed iPSC propensity and intrinsically increased differentiation toward the parental cell lineage (10). In particular, the intrinsic

\section{Related Commentary: p. 4331}

Conflict of interest: The authors have declared that no conflict of interest exists. Submitted: May 8, 2015; Accepted: September 24, 2015.

Reference information: / Clin Invest. 2015;125(12):4463-4482. doi:10.1172/JCI82735. myogenic propensity observed in reprogrammed mesoangioblasts (MABs) (11) might prove useful in driving cell fate in the context of skeletal muscle repair. Also, analogous findings have recently been reported in the context of cardiac epigenetic memory (10). However, it is still unknown whether the source-related myogenic propensity influences the switch between cardiac and skeletal myogenic lineages. Moreover, it is still an open question whether such differential propensity would affect the combined regeneration of both striated muscle types in vivo.

In this study, we addressed the combined treatment of striated muscles by conjugating the iPSC myogenic propensity with the prospective isolation of mesodermal iPSC-derived progenitors (MiPs) in isogenic settings of murine, canine, and human cells.

\section{Results}

Differential myogenic propensity influences iPSC-based chimerism in fetal and adult tissues. To exclude interferences caused by genetic background or unrelated individual variability, we reprogrammed murine iPSCs from isogenic fibroblasts (f-iPSCs) and MABs (MAB-iPSCs), both isolated from syngeneic male mice (Supplemental Figure 1, A and B; supplemental material available online with this article; doi:10.1172/JCI82735DS1). Isogenic $\mathrm{f}$ - and MAB-iPSCs displayed a normal karyotype and comparable expression levels of pluripotency markers (Supplemental Figure 1C). In contrast, a teratoma assay showed a higher differentiation propensity of 

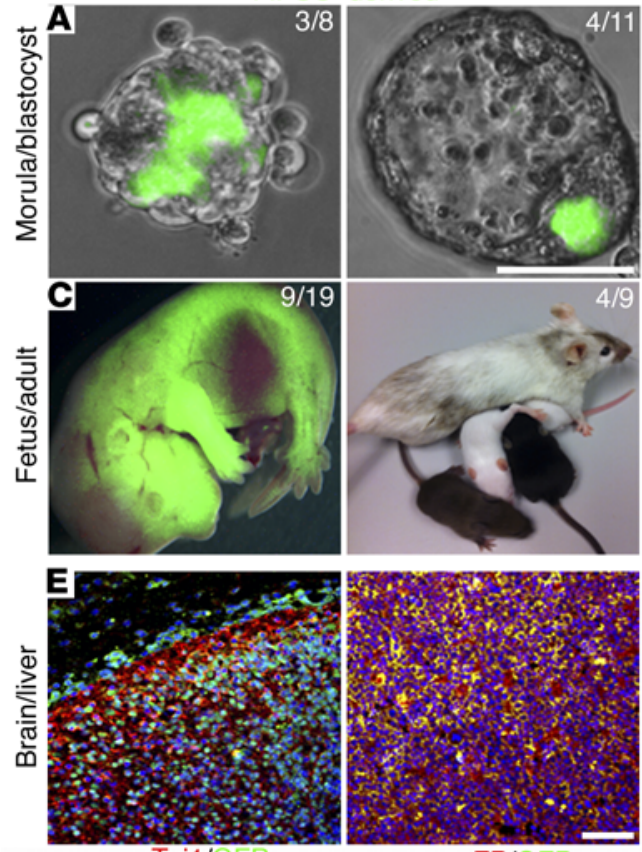

Tuj1/G
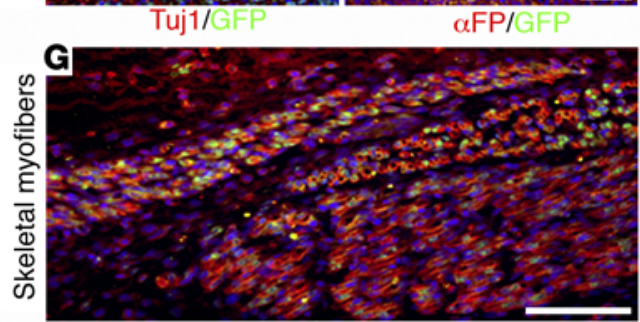

Sarc $\alpha$ Act/
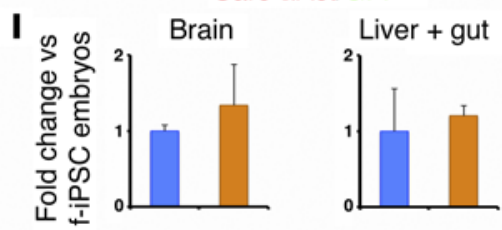
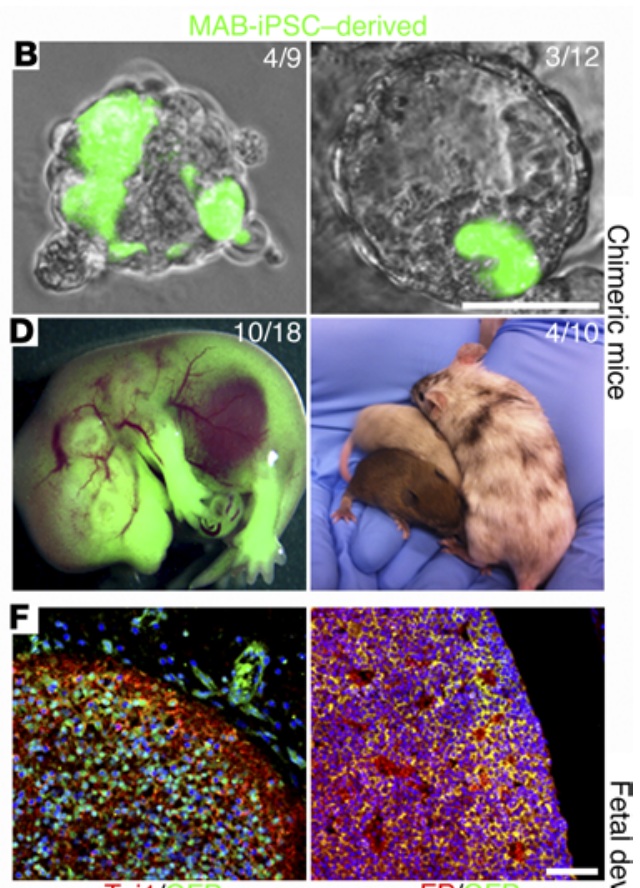

Tuj1/G

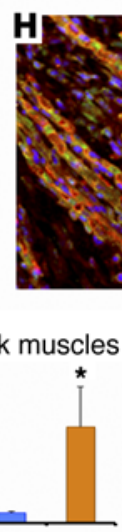

Figure 1. Analysis of myogenic propensity of iPSCs in fetal chimeric tissues. (A-H) Isogenic GFP' $f$ - and MAB-iPSCs contributed at comparable rates to chimeric morulae and blastocysts ( $\mathbf{A}$ and $\mathbf{B}$ ) and to chimeric fetuses (E14.5) and fertile adults (C and $\mathbf{D})$. Rates of GFP+ ${ }^{+}$chimeric embryos are reported for each stage ( $n=5 /$ iPSC line). However, immunofluorescence analysis of fetal tissues at E14.5 revealed comparable levels of GFP contribution to brain and liver ( $\mathbf{E}$ and $\mathbf{F}$ ) and unequal contribution levels (higher for MAB-iPSCs) to nascent somitic myofibers ( $\mathbf{G}$ and $\mathbf{H}$ ) ( $n=6$ embryos/iPSC type for fetal tissue analyses). Scale bars: $\sim 50 \mu \mathrm{m}$ (A and B) and $\sim 00 \mu \mathrm{m}$ (C-H). (I) At E14.5, qPCR analyses of dissected tissues from chimeric fetuses showed significantly higher GFP expression levels in the trunk muscles of MABiPSC chimeric fetuses compared with levels detected in $\mathrm{f}$-iPSC chimeric fetuses $\left({ }^{*} P<0.05, n \geq 3 /\right.$ iPSC type, Mann-Whitney $U$ test). Error bars represent SD. $\alpha \mathrm{FP}, \alpha$-fetoprotein.
MAB-iPSCs toward the skeletal muscle lineage compared with that of f-iPSCs (Supplemental Figure 1D), thus confirming that we had established an isogenic setting of differential myogenic propensity.

To test the impact of iPSC myogenic propensity on tissue development, we asked whether f- and MAB-iPSCs differentially contribute to chimeric tissues after morula aggregation. We found that both $\mathrm{GFP}^{+} \mathrm{f}$ - and MAB-iPSCs contributed to tissues of chimeric embryos and fertile adults, which displayed variable chimerism in coat color and germline transmission (Figure 1, A-D). When assaying the germ layer derivatives during development, MAB-iPSCs contributed to a similar extent to fetal brain and liver (Figure 1, $\mathrm{E}$ and $\mathrm{F}$ ), but contributed to a significantly greater extent to the nascent skeletal muscle fibers as compared with f-iPSCs (Figure $1, \mathrm{G}-\mathrm{I})$. In the adult tissues, we observed a greater contribution of MAB-iPSCs to the postnatal skeletal muscles of chimeric mice in both the absence and presence of cardiotoxin-induced regeneration (Figure 2, A-C). We then asked whether the iPSC-specific contribution to the resident pools of myogenic stem cells was different. In accordance with the results observed in adult chimeric muscles, we found a higher $\mathrm{GFP}^{+}$fraction of freshly isolated satellite cells and MABs in MAB-iPSC-chimeric mice than in f-iPSC-chimeric mice during both proliferation and differentiation stages (Figure 2, D-F). Intriguingly, the $\mathrm{GFP}^{+}$contribution to the adult myocardium appeared robust and comparable between f- and MAB-iPSCs (Figure 2, G and H). Also, other nonmesodermal postnatal tissues, including liver and skin, did not show significant differences in chimerism under steady state and after injury (Supplemental Figure 2). Thus, the differential myogenic propensity did not affect the pluripotency of MAB-iPSCs, but selectively enhanced in vivo chimerism in fetal and adult skeletal muscles. In addition, the myogenic bias did not influence iPSCdriven chimerism in the adult cardiac muscle.

MiPs retain their differential myogenic propensity. In order to address the question of whether the myogenic propensity would impact the regeneration of striated muscles, we sought a novel progenitor pool that would be amenable to the regeneration of both striated muscle types in vivo. To this end, we developed a prospective sorting strategy for differentiating iPSCs. This approach was based on myogenic mesoderm induction (12) and isolation of cells that express CD140a, CD140b, and CD44 (Figure 3, A and B), sur- 
A
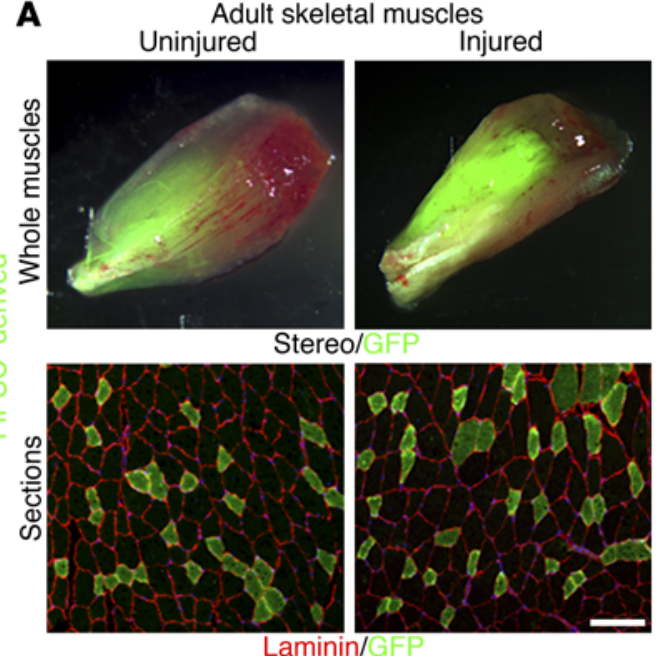

B
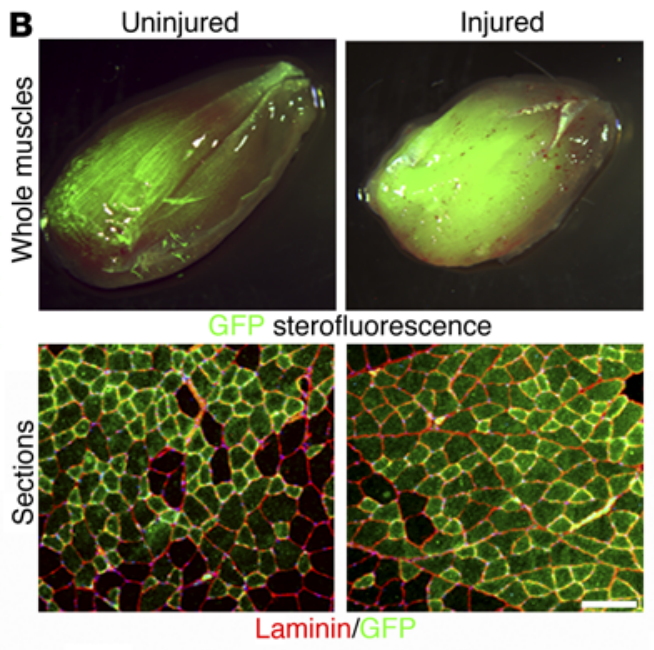

C

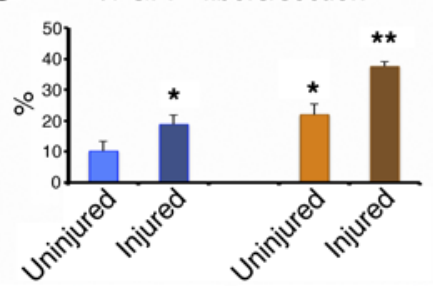

D

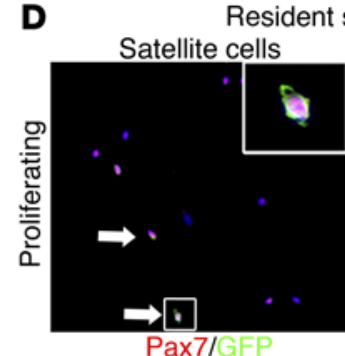

Resident

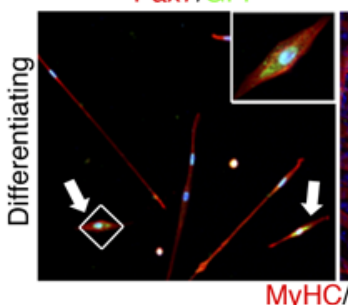

$\mathrm{MyHCl}$

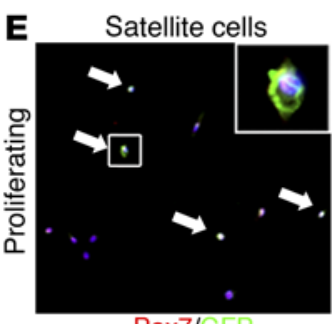

Pax7/C

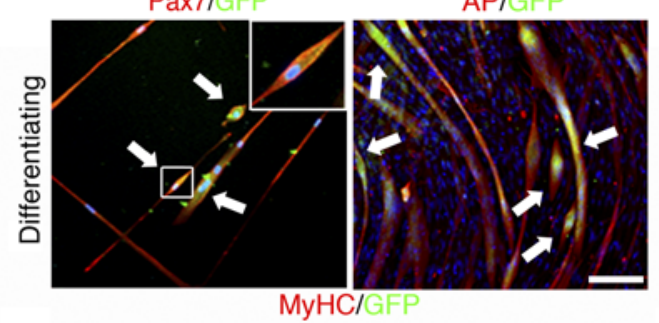

$\mathbf{F}$

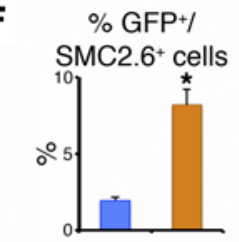

f-iPSC-chimeric mice
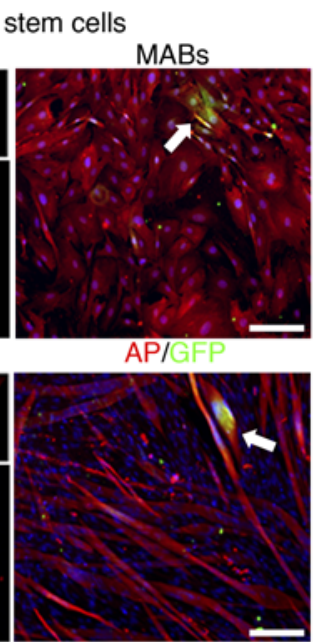

MABs

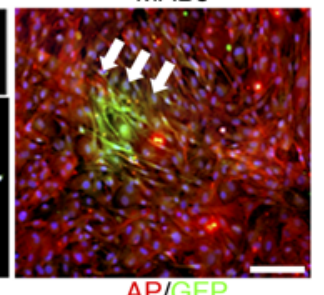

Primary isolation

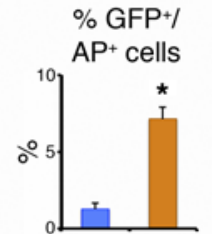

MAB-iPSC-chimeric mice
G Heart

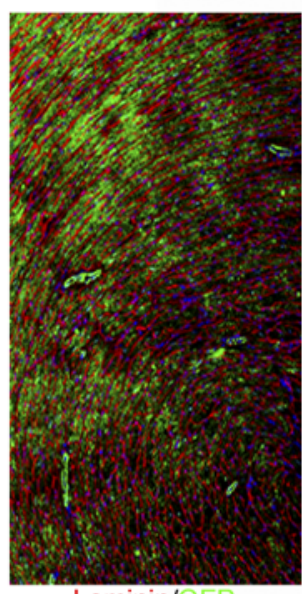

Laminin/C

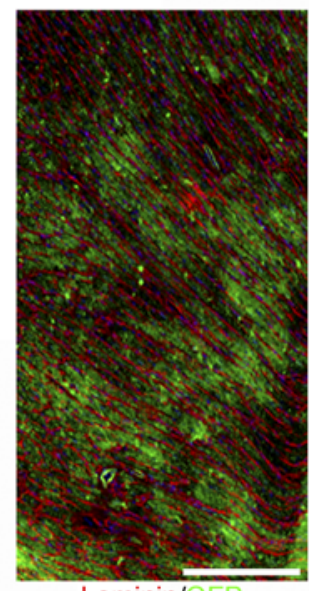

Laminin/C

H $\%$ GFP $^{+}$areas/

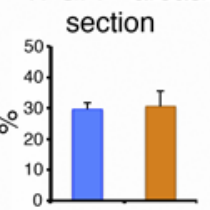

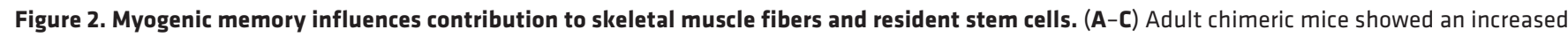

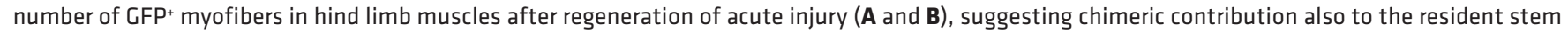
cell pools. In addition, MAB-iPSC-chimeric mice displayed a greater chimeric contribution in both conditions as compared with f-iPSC-chimeric mice (C). (D and E) Once purified from the uninjured muscles, primary pools of satellite cells and MABs appeared chimeric at passage 0 , with larger GFP+ subfractions (arrows) in the cell pools from MAB-iPSC mice, in both proliferation (D) and differentiation (E) stages. Quantitative difference in the GFP+ subfractions was confirmed by cytometry (F). (G-N) In contrast, MAB-iPSCs and f-iPSCs did not apparently differ in the GFP+ contribution to the myocardium (G-H). $n=4$ mice/iPSC type. ${ }^{*} P<0.05$ versus uninjured f-iPSC mice; ${ }^{*} P<0.05$ versus injured f-iPSC mice, Kruskal-Wallis and Mann-Whitney $U$ tests. Error bars represent SD. Original magnification, $\times 40$ (insets) of selected areas; scale bars: $\sim 100 \mu \mathrm{m}$.

face markers shared by somatic stem cells from cardiac and skeletal muscles (2). When compared with the negative fraction, $\mathrm{CD} 140 \mathrm{a}^{+} \mathrm{CD} 14 \mathrm{Ob}^{+} \mathrm{CD} 44^{+}$cells showed enrichment in gene and $\mathrm{miR}$ markers of myogenic mesoderm transition (Figure 3B and Supplemental Figure 3, A and B), including Mesp1 (13) and Mir133a (14). Therefore, we named these cells MiPs. We then compared the ability of MiPs to differentiate toward striated muscle lineages in vitro when derived from isogenic $\mathrm{f}$ - and MAB-iPSCs (f-MiPs and MAB-
MiPs, respectively). Intriguingly, the commitment of $\mathrm{f}$ - and MABMiPs to the cardiomyogenic lineage appeared robust and comparable, both in coculture with beating neonatal cardiomyocytes and after differentiation into immature cardiomyocyte-like cells (Figure 3C, Supplemental Videos 1-4, and Supplemental Figure 3C). Conversely, MAB-MiPs showed a significantly higher propensity for skeletal muscle differentiation than did f-MiPs, both in coculture with myoblasts (Figure 3C) and after a pulse of Pax3 and Pax7 over- 

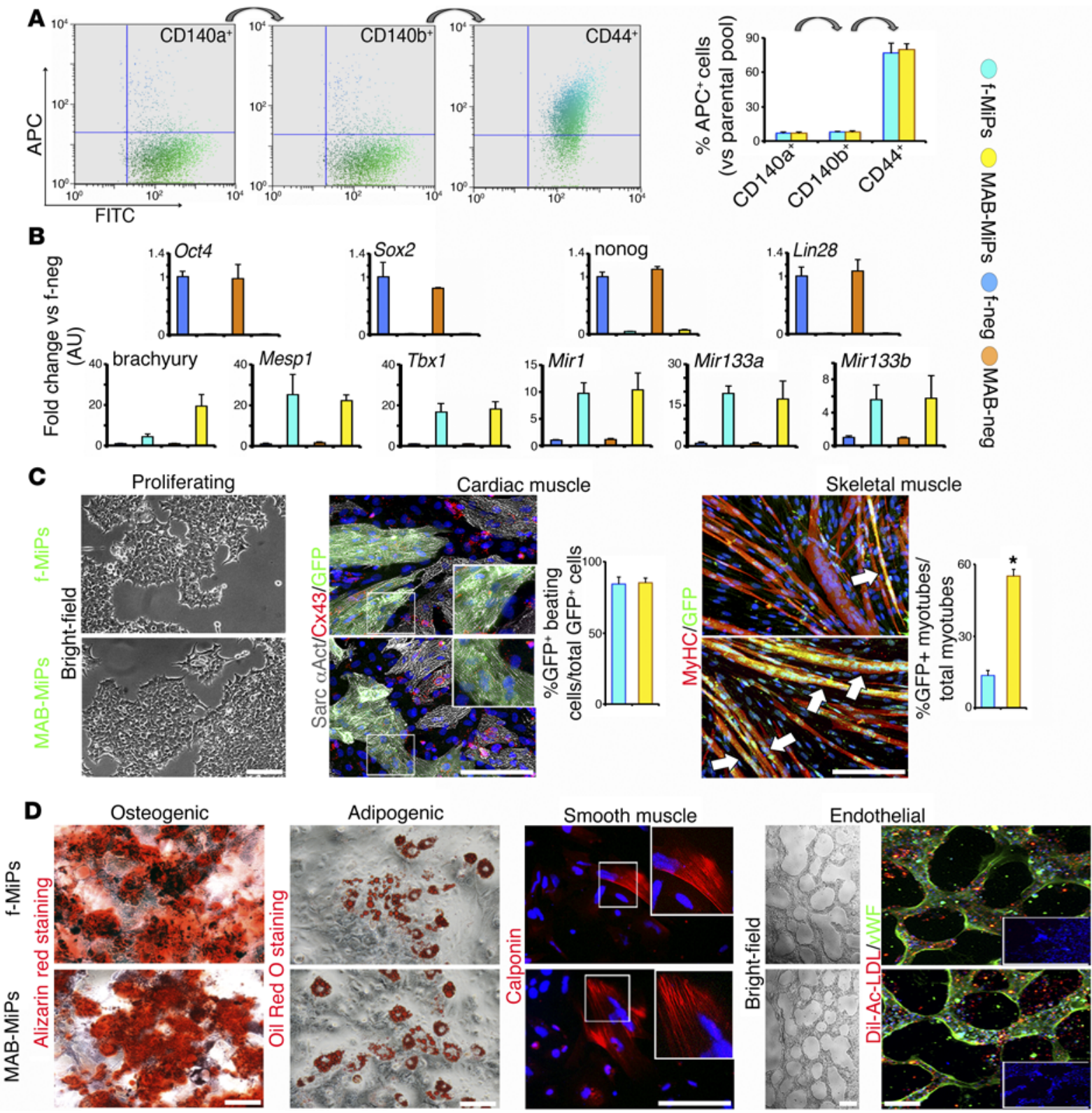

Figure 3. Isolation and characterization of murine MiPs. (A) MiPs were isolated by triple sequential sorting for CD140a, CD140b, and CD44 from GFP ${ }^{+}$ iPSCs with respect to both MiP ( $f$ - and MAB-MiPs) and negative ( $f$-neg and MAB-neg) pools. At all steps, positive fraction yields were quantitatively comparable between $\mathrm{f}$ - and MAB-derived MiPs. (B) SYBR Green- and TaqMan-based qPCR analyses showed that pluripotency factors (Oct4, Sox2, nanog, and Lin28) were downregulated, whereas gene and miR markers of mesodermal transition and maturation (brachyury, Mesp1, Tbx1, Mir1, Mir133a, and Mir133b) were upregulated in both $\mathrm{f}$ - and MAB-MiPs compared with the negative pools ( $n=3 / \mathrm{MiP}$ type). Error bars represent SD. (C) GFP+ MiPs had comparable morphologies during proliferation and differentiated into beating cardiomyocyte clusters in combination with neonatal rat cardiomyocytes at comparable rates (original magnification, $\times 40$ for insets of selected areas, showing a mature Sarc $\alpha$ Act pattern). Intriguingly, after coculture with myoblasts, MABMiPs resulted in higher numbers of GFP+ ${ }^{+}$yotubes (arrows) as compared with f-MiPs. $n=4 /$ MiP type. ${ }^{*} P<0.05$ versus f-MiPs, Mann Whitney $U$ test. Error bars represent SD. (D) Under opportune stimulations in vitro, both $\mathrm{f}$ - and MAB-MiPs showed the capacity to undergo osteogenic (alizarin red staining), adipogenic (Oil Red $\mathbf{O}$ staining), smooth muscle (immunofluorescence staining for calponin; original magnification, $\times 40$ for insets of selected areas, showing partial fibrillar organization), and endothelial differentiation (tubular structures formed on a Geltrex layer; left panels) and were characterized by deposition of endothelium-specific extracellular matrix (von Willebrand factor [VWF]) and uptake of Dil-Ac-LDL at comparable rates (insets show the negative staining control and proliferating cells at equal magnification). $n=4 / \mathrm{MiP}$ type. Scale bars: $\sim 100 \mu \mathrm{m}$.

expression (Supplemental Figure 3D). In addition, $\mathrm{f}$ - and MAB-MiPs differentiated to a comparable extent toward other mesodermal lineages such as osteogenic, adipogenic, smooth muscle, and endothelial lineages (Figure 3D). Considering the different behavior in the two striated muscle lineages, we analyzed the expression pattern of key markers during iPSC-to-MiP differentiation and in isolated f- versus MAB-MiPs. Quantitative PCR (qPCR) analyses showed that markers of pluripotency (nanog, Oct4) were comparably downregulated over time (Supplemental Figure 3E). Markers of mesodermal transition (brachyury, Meox1, Mixl1) were not found to be differ- 
A
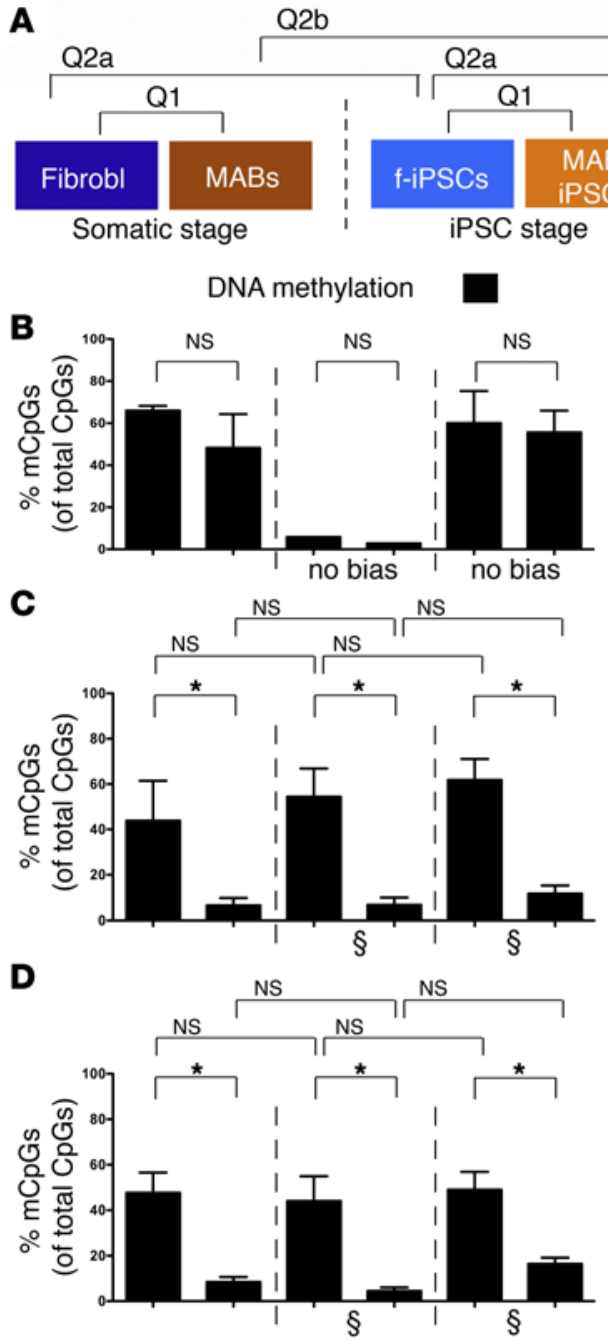

E

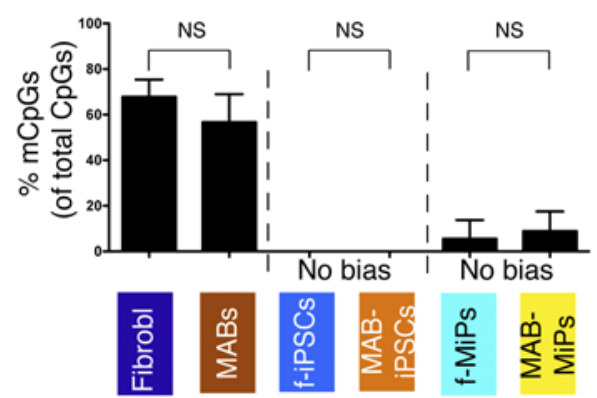

Q2b

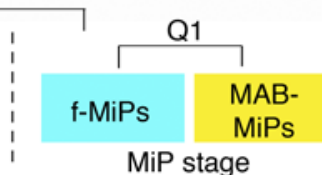

Statistical score of progeny-related bias at IPSC and MiP stages:

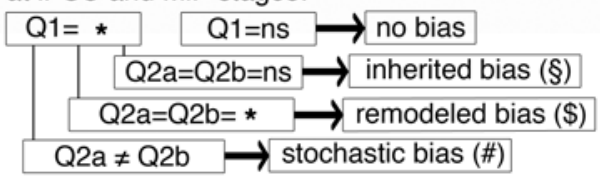

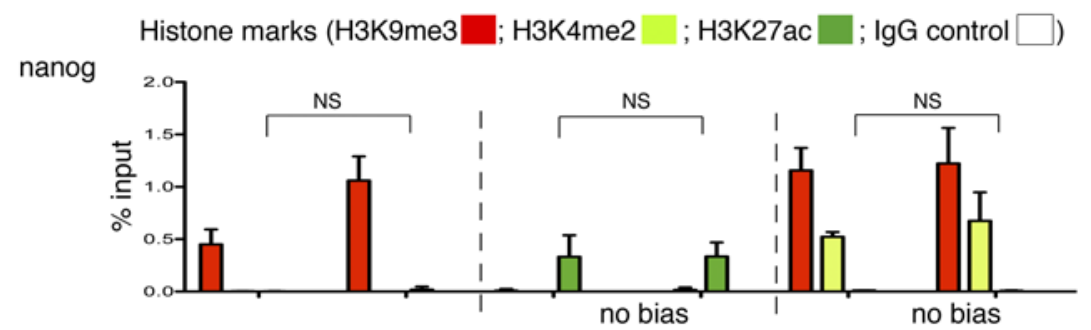

brachyury

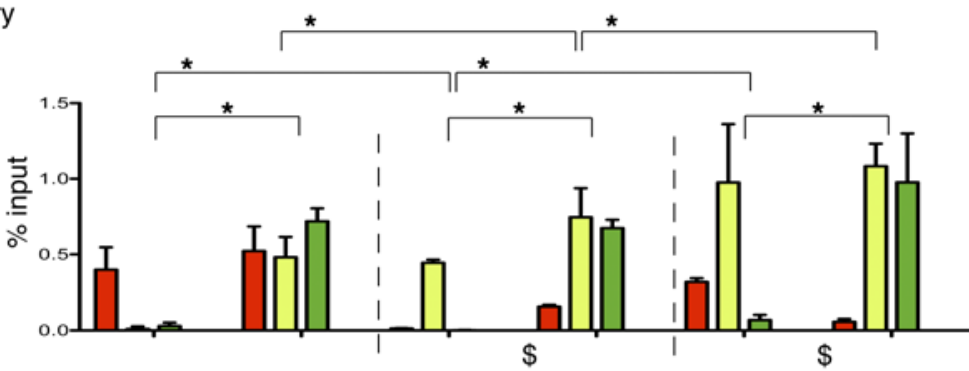

Pax7

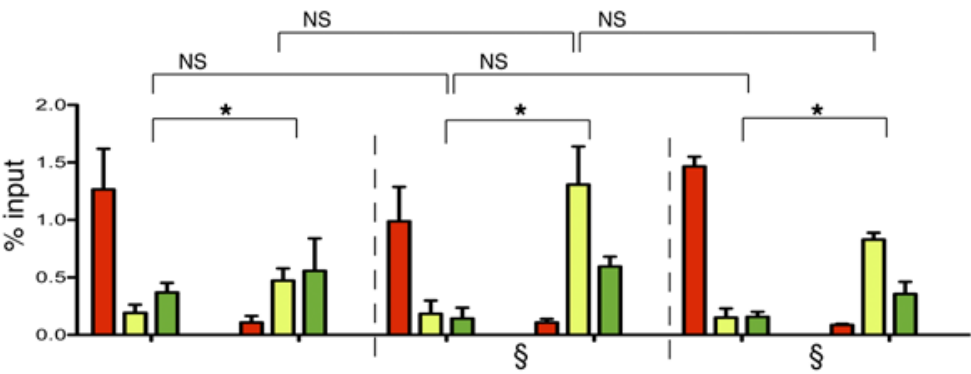

Tbx5

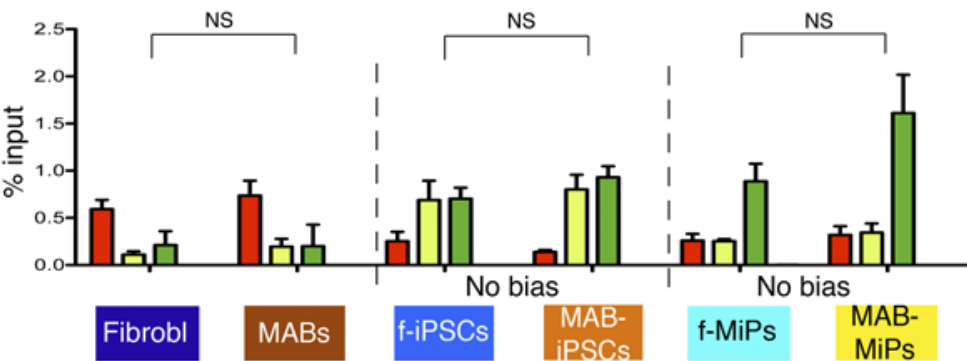

Figure 4. Differential myogenic propensity correlates with specific epigenetic signatures. (A) At both iPSC and MiP stages, we compared the quantitative epigenetic data (methylation percentage by 1-way ANOVA and histone mark enrichment by 2-way ANOVA) of f-and MAB-derived cells in specific loci using a hierarchical system of three queries. Query 1 (Q1) addressed the difference between $\mathrm{f}$ - and MAB-derived cells within the same stage. When Q1 = *, we addressed the difference between each cell population and the related parental cells within the same progeny (Q2a, f progeny; Q2b, MAB progeny). When $\mathrm{Q} 2 \mathrm{a}=\mathrm{Q} 2 \mathrm{~b}=\mathrm{NS}$, the bias was considered inherited $(\S)$; when $\mathrm{Q} 2 \mathrm{a}=\mathrm{Q} 2 \mathrm{~B}={ }^{*}$, the bias was considered remodeled (\$); finally, in cases of nonunivocal results $(\mathrm{Q} 2 \mathrm{a} \neq \mathrm{Q} 2 \mathrm{~b}$ ), the bias was considered stochastic (\#). This statistical categorization was applied to both bisulphite sequencing (DNA methylation; data expressed as a percentage of methylated CpGs) and ChIP-qPCR data (H3K marks; data expressed as a percentage of input) of $\mathrm{CpC}$ islands of target genes. (B) Following this statistical model, the pluripotency marker nanog showed stage-specific changes in epigenetic cues, with no progeny-related bias (Q1 = NS). (C) The mesodermal marker brachyury showed inherited, progeny-related bias in methylation ( $\S$ ), but remodeled biases in histone marks (\$). MAB-iPSCs and MAB-MiPs showed more permissive/activating epigenetic cues (lower methylation, higher levels of H3K4me2 and H3K27ac). (D) The skeletal myogenesis marker Pax7 presented inherited biases in both methylation and histone marks at both iPSC (§) and $\mathrm{MiP}(\S)$ stages. MAB-derived cells showed a durable bias in lower methylation and permissive/activating histone marks. (E) Conversely, the cardiac myogenesis marker Tbx5 showed stage-specific shifts in methylation and histone marks, with no significant progeny-related bias (Q1 = NS). Both MiP types showed low methylation levels and enrichment in H3K27ac. $n=3$ /cells pool. ${ }^{*} P<0.05$, 1-way ANOVA with Bonferroni's multiple comparisons test for DNA methylation analysis; 2-way ANOVA with Bonferroni's multiple comparisons test for histone mark analysis. All analyses included data from isogenic clones from 3 syngeneic individuals. fibrobl, fibroblasts; $\mathrm{mCpGs,} \mathrm{methylated} \mathrm{CpGs.}$ 
A

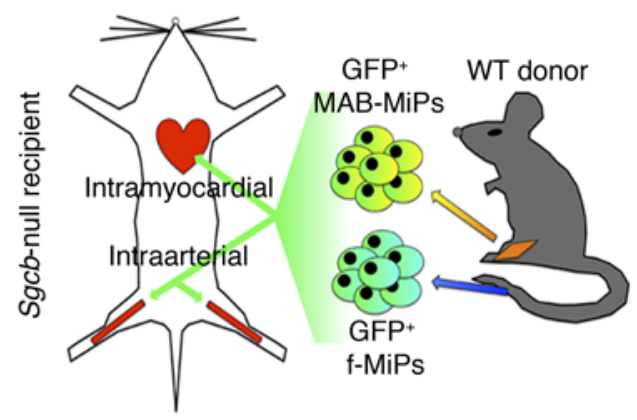

Sham
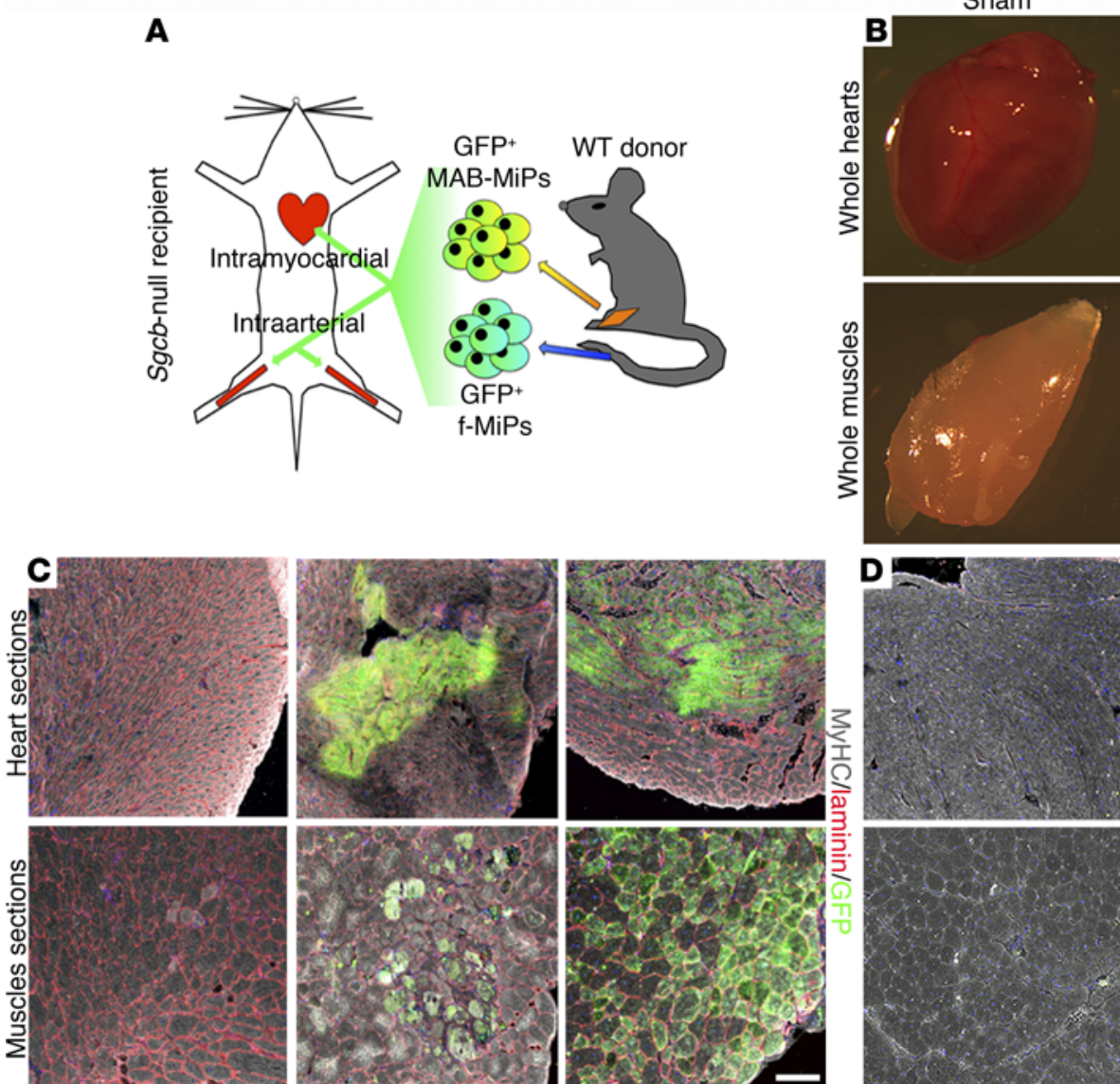
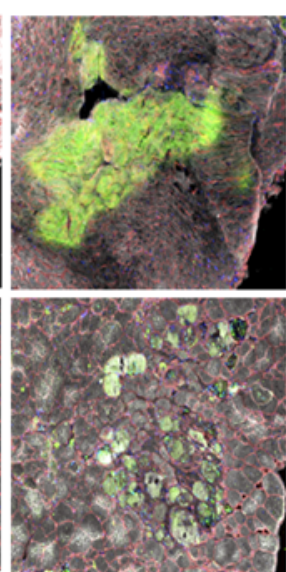
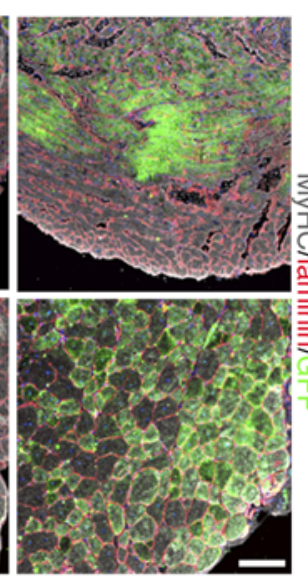

\section{D}

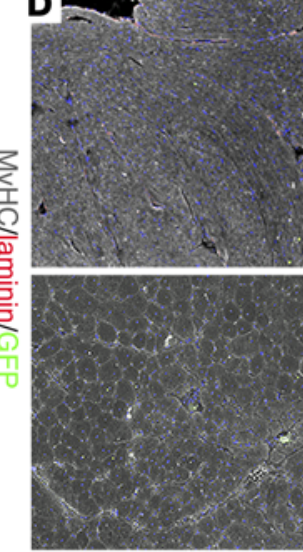

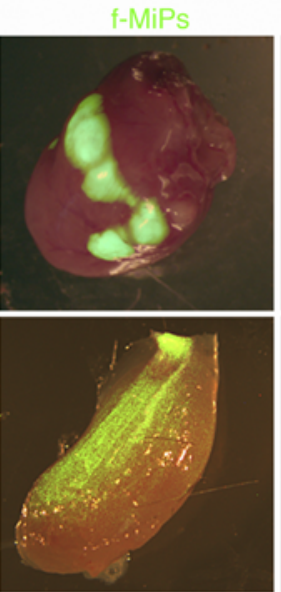
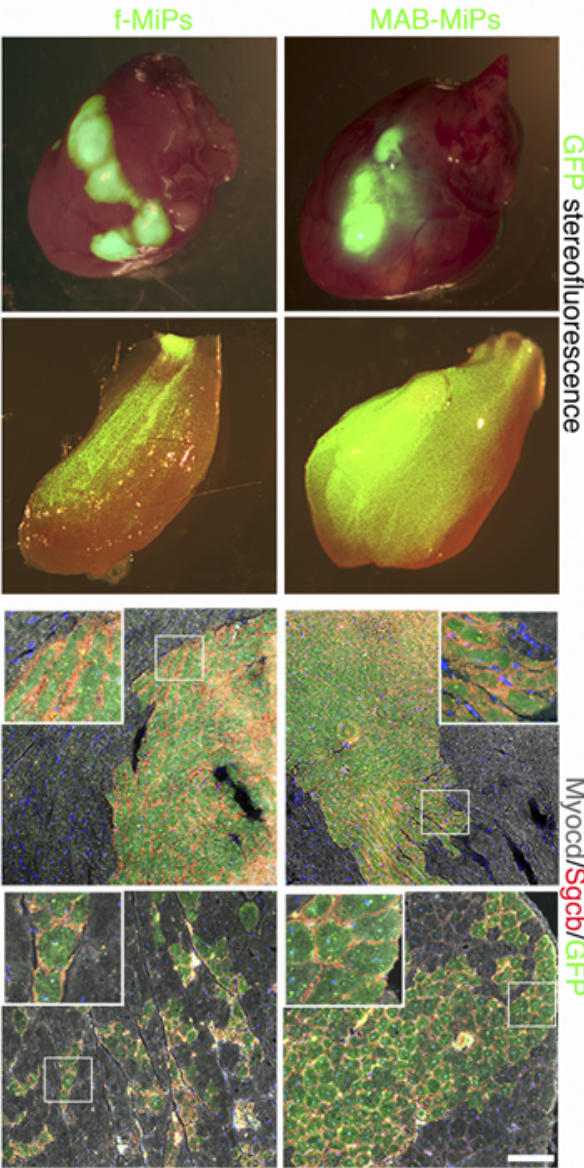

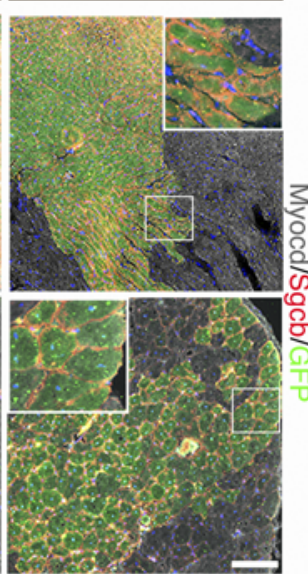

Figure 5. In vivo combined regeneration of cardiac and skeletal muscles. (A) Schematic representation of the parallel delivery system of GFP ${ }^{+} f-$ and MAB-MiPs injections into the myocardium and both femoral arteries of Sgcb-null dystrophic cardiomyopathic mice 3 months after birth. Four weeks after injection, both $\mathrm{f}$ - and MAB-MiPs comparably engrafted the myocardium, whereas MAB-MiPs engrafted the hind limb muscles (shown are tibialis anterior muscles) markedly more than did f-MiPs, as shown by stereofluorescence (B) and immunostaining (C). (D)Accordingly, immunofluorescence analysis showed comparable numbers of GFP+ $\mathrm{Sgcb}^{+}$cardiomyocytes in the heart, whereas a markedly greater number of GFP+ $S g c b^{+}$fibers were observed in MAB-MiP-treated muscles compared with those in $\mathrm{f}$-MiP controls (gastrocnemius muscles are shown in $\mathbf{D}$ ). $n=8$ mice/group.

entially patterned (with the exception of Mixl1) during bulk differentiation, but were found to be differentially expressed in isolated MiPs (Supplemental Figure 3F). In contrast, markers of skeletal myogenesis (Pax3, Pax7, Desm) were differentially patterned during iPSC differentiation and at the MiP stage (Supplemental Figure $3 G)$. Conversely, expression levels of cardiac myogenesis markers (Tbx5, Gata4, Flk1) appeared comparable during differentiation and in isolated MiPs (Supplemental Figure 3H). Consistent with the notion of epigenetic memory (15), we sought to determine whether the differential myogenic propensity correlated with progeny-specific epigenetic cues across somatic, iPSC, and MiP stages. We focused our analyses on DNA CpG methylation and on histone 3 lysine (H3K) marks - specifically, trimethylation of lysine 9 (H3K9me3), dimethylation of lysine 4 (H3K4me2), and acetylation of lysine 27 (H3K27ac). DNA CpG methylation is a broadly investigated trait for epigenetic regulation (16). $\mathrm{H} 3 \mathrm{~K} 9 \mathrm{me} 3$ is a repressive histone marker associated with bias retainment and differentiation in striated muscle cells $(17,18)$. H3K4me 2 marks a permissive state for genes promoting both skeletal (19) and cardiac (20) myogenic lineages, and the $\mathrm{H} 3 \mathrm{~K} 9 \mathrm{me} 3 / \mathrm{H} 3 \mathrm{~K} 4 \mathrm{me} 2$ ratio on specific promoters has been associated with clinical parameters of facioscapulohu- meral MD (21). H3K27ac has been described as a predictive activating marker for CpG-related promoters in muscle cells as well (22). Given the previous indications that epigenetic biases are located in key upstream promoters in the context of intrinsic myogenic propensity (11), we narrowed our analyses of DNA methylation and H3K signature patterns to the CpG islands upstream of the transcription start sites of the aforementioned key markers. Overall, parental somatic cells exhibited a prevalently "closed" (high methylation, prevalence of $\mathrm{H} 3 \mathrm{~K} 9 \mathrm{me} 3$ ) state for pluripotency and cardiomyogenic markers, whereas MAB progenies showed a significantly more "open" (low methylation, prevalence of H3K4me2 or H3K27ac) state for skeletal myogenic genes and brachyury (Figure 4 and Supplemental Figures 4-6). We then applied a hierarchical statistical system to quantitatively assess the progeny-related retainment of specific patterns of methylation and H3K marks across iPSC and MiP stages. First, the epigenetic patterns were compared between $\mathrm{f}$ - and MAB-iPSCs and between $\mathrm{f}$ - and MAB-MiPs (query 1 [Q1]). Then, the epigenetic patterns were compared between stage-specific and parental cells (Q2) for both progenies (Q2a, f-derived cells; Q2b, MAB-derived cells). When the epigenetic trait pattern was significantly different between $f$ - and 
A
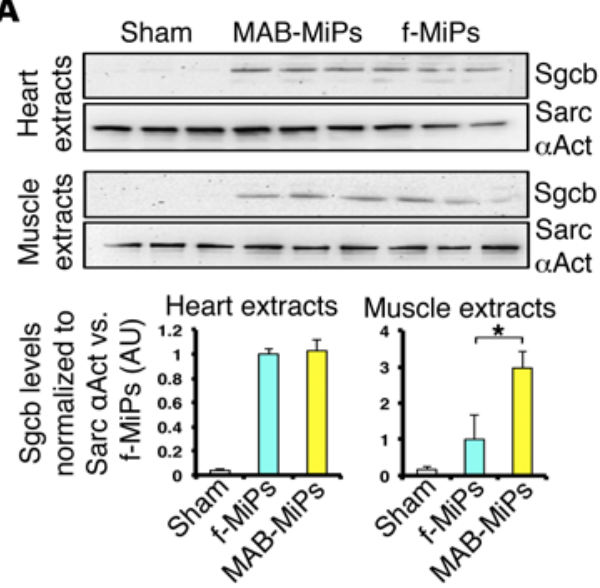

B

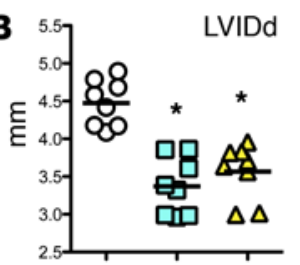

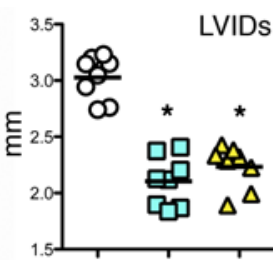
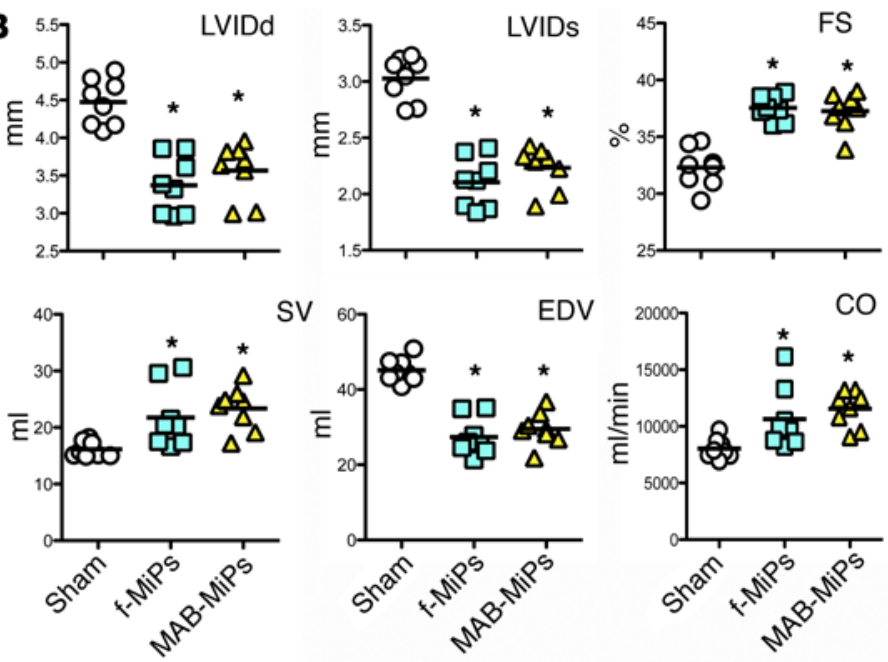

D
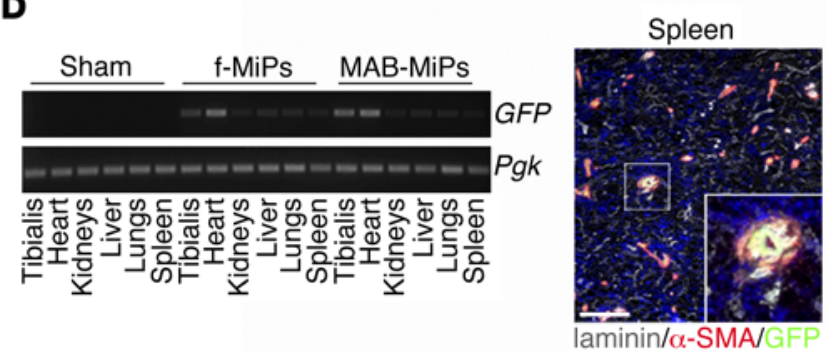

C
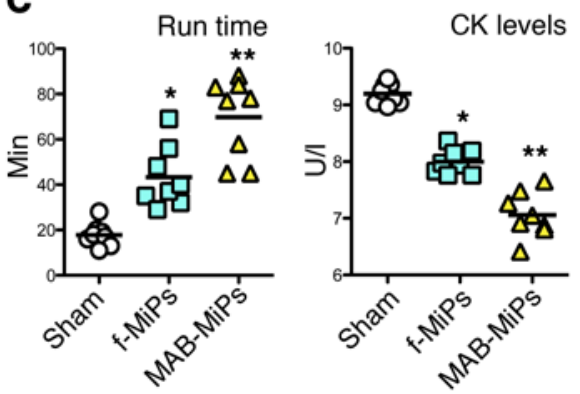

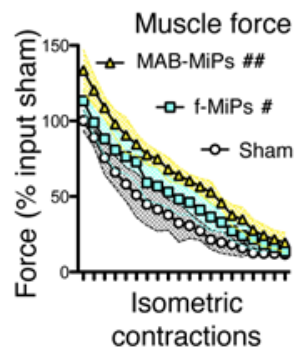

contractions

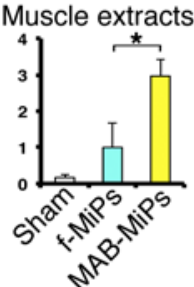

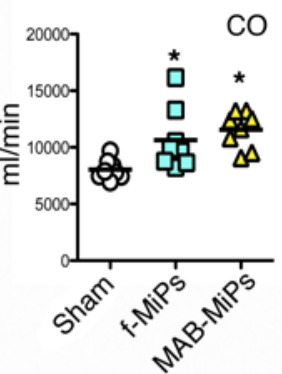

$\mathrm{CO}$

Figure 6. Functional regeneration of skeletal, but not cardiac, muscles is influenced by MiP myogenic propensity. (A) WB and densitometric analyses of Sgcb protein in MiP-injected cardiac and skeletal muscle 4 weeks after injection. Consistent with the immunofluorescence data, Sgcb levels were comparable in MiP-treated myocardium samples and significantly higher in MAB-MiP-treated skeletal muscle (tibialis anterior) biopsies. $n=3 /$ group. ${ }^{*} P<0.05$, Mann-Whitney $U$ test. Error bars represent SD. (B) 3D echocardiographic results showed cardiac functionality that was significantly and comparably ameliorated by $\mathrm{f}$ - and MAB-MiPs. (C) In contrast, the amelioration in treadmill performance (run time) and serum CK levels was markedly increased in MAB-MiP- versus f-MiP-treated mice. $n=8$ mice/group. ${ }^{*} P<0.05$ versus sham; ${ }^{*} P<0.05$ versus sham and $\mathrm{f}$-MiPs, Kruskal-Wallis and Mann-Whitney $U$ tests. Each data point refers to 1 animal. Error bars represent average values. Accordingly, 8 weeks after injection, the absolute force measurement in EDL muscles under iterated bouts of isometric contraction showed significant improvement in the force curve in $\mathrm{f}$-MiP-injected versus sham-injected mice, and in MAB-MiP- versus $\mathrm{f}-\mathrm{MiP}$-injected mice. $n=5$ mice/group. ${ }^{P} P<0.05$ versus sham; ${ }^{\# P}<0.05$ versus sham and $\mathrm{f}-\mathrm{MiPs}$, 2-way ANOVA. Data represent average values expressed as a percentage of input sham (average value at approximately 1 for sham muscles); ribbons represent the interval of SD. (D) qPCR analysis of heart, skeletal muscles, and filter organs showed barely detectable GFP signal in liver, lungs, spleen, and kidneys 8 weeks after delivery of $\mathrm{f}$ - and MAB-MiPs. Immunofluorescence analysis revealed the occasional occurrence (generally $\leq 2$ per section) of $\mathrm{GFP}^{+} \alpha-\mathrm{SMA}^{+}$small vessels in filter organs of MiP-treated animals (inset shows the spleen of an f-MiP-injected animal; $n=5$ mice/cohort), suggesting very limited levels of off-target engraftment. Insets, $\times 40$ magnification of selected areas; scale bars: $100 \mu \mathrm{m}$.

$\mathrm{MAB}$-derived cells at the $\mathrm{iPSC}$ or MiP stage $\left(\mathrm{Q} 1=^{*}\right)$, we further set Q2 conditions to score whether the bias was retained or remodeled from the parental cell stages, or stochastically introduced during stage-to-stage cell transition. When the bias was not significantly different from that of the parental cells in both progenies $(\mathrm{Q} 2 \mathrm{a}=$ $\mathrm{Q} 2 \mathrm{~b}=\mathrm{NS})$, the bias was considered inherited $(\S)$; whereas it was considered remodeled (\$) when the bias was significantly different from that of the parental cells in both progenies $\left(\mathrm{Q} 2 \mathrm{a}=\mathrm{Q} 2 \mathrm{~b}={ }^{*}\right)$. When the comparison with the parental cells was not univocal between progenies $(\mathrm{Q} 2 \mathrm{a} \neq \mathrm{Q} 2 \mathrm{~b})$, the bias was considered stochastic (\#) (Figure 4A). According to bisulphite sequencing (DNA methylation levels) and ChIP-qPCR (H3K mark enrichment) analyses, the epigenetic state of nanog and Oct4 appeared, as expected, to be open in iPSCs and partially closed in MiPs, with no bias discriminating between $\mathrm{f}$ - and MAB-derived progenies $(\mathrm{Q} 1=\mathrm{NS}$; Figure $4 \mathrm{~B}$ and Supplemental Figure 5A). Interestingly, mesodermal transition markers showed nonunivocal trends in epigenetic regulation. Indeed, analysis of brachyury showed an inherited bias in DNA methylation and a remodeled bias in histone marks in both iPSCs and MiPs (Figure 4C). Meox1 showed progeny-related biases only in DNA methylation that were inherited in iPSCs and remodeled in MiPs (Supplemental Figure 5B). Mixl1 showed remodeled biases in H3K marks at both stages (Supplemental Figure 5C). In line with skewed gene expression regulation, the skeletal myogenic markers Pax7, Pax3, and Desm were characterized by inherited biases in DNA methylation and H3K marks at both iPSC and MiP stages (Figure 4D and Supplemental Figure 5, D and E). Conversely, the epigenetic state of Tbx5, Gata4, and Flk1 appeared open in iPSCs and MiPs, with no bias discriminating against $\mathrm{f}$ - and MAB-derived progenies (Q1 = NS; Figure 4E and Supplemental Figure 6, A and B). Considering the difference in expression and the inherited epigenetic biases for brachyury and skeletal myogenic genes such as 
A
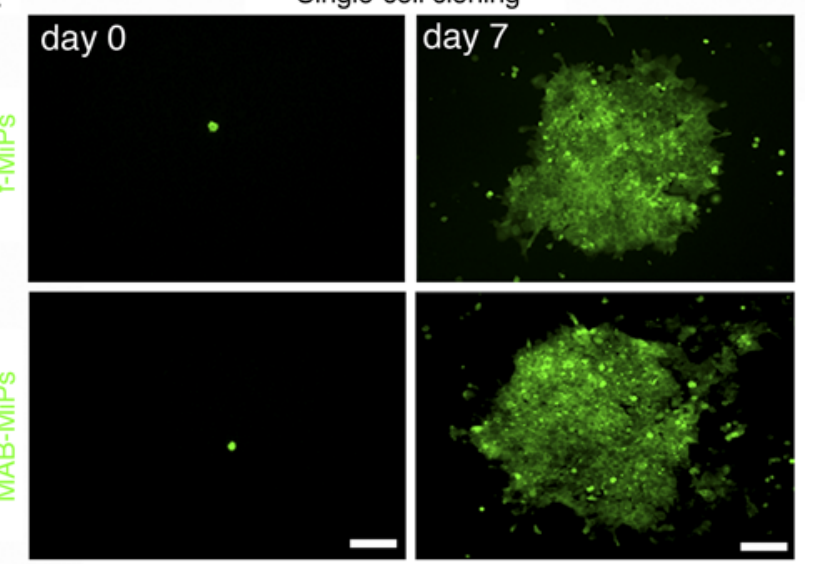

B
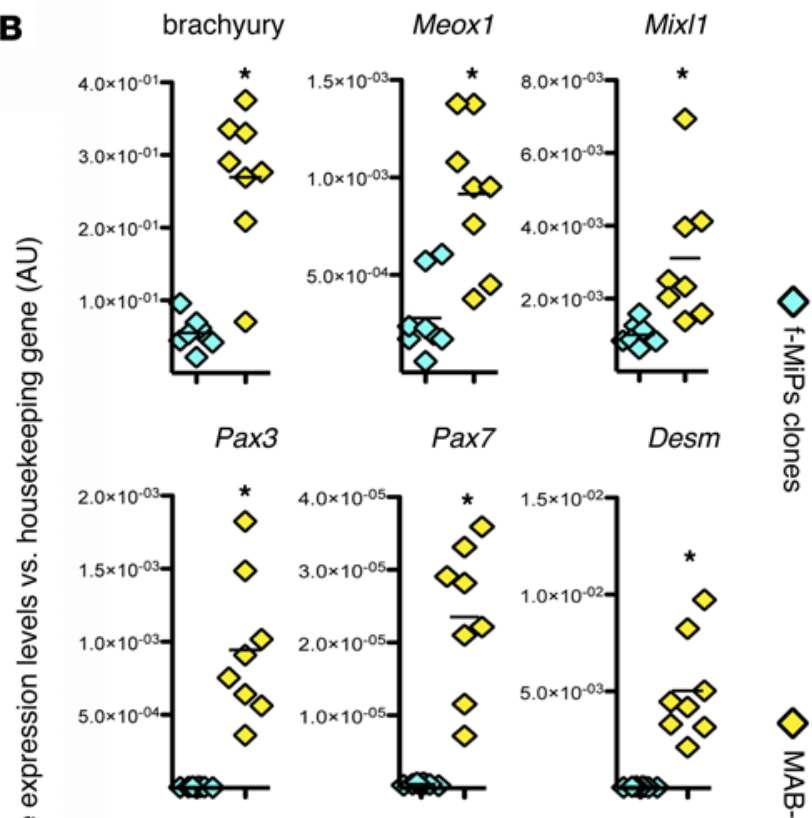

Tbx5

Gata4
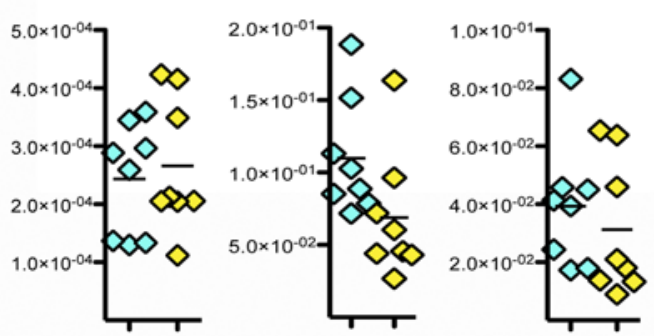

Figure 7. Single-cell clone characterization of $f-$ and MAB-MiPs. (A) Murine $\mathrm{GFP}^{+} \mathrm{MiPs}$ were single-cell cloned by limiting dilution. (B) qPCR analysis showed significant upregulation of mesodermal markers (brachyury, Meox1, Mix/1) and skeletal myogenic markers (Pax7, Pax3, Desm), but no significant differences in cardiomyogenic markers ( $T b \times 5$, Gata4, Flk1), recapitulating what was observed in whole-cell pools. Data represent relative expression values (absolute expression normalized to the housekeeping gene), and each data point refers to the average value of 1 clone; $n=8$ clones/MiP type. ${ }^{*} P<0.05$, unpaired $t$ test.
Pax7, we asked whether restoring the expression of these genes in f-MiPs to levels comparable to those in MAB-MiPs induces a corresponding increase in myogenic propensity. We optimized transfection conditions to pulse f-MiPs with transient overexpression of both genes to levels similar to those in control MAB-MiPs (Supplemental Figure $6 \mathrm{C}$ ), and we then assessed their myogenic propensity in coculture with myoblasts or via spontaneous differentiation. While overexpression of brachyury did not induce significant effects and Pax7 pulsing induced only limited effects, we found, intriguingly, that the combination of both gene pulses significantly ameliorated the myogenic propensity of f-MiPs to levels similar to those detected in control MAB-MiPs (Supplemental Figure 6, D and E). As expected, pulsing MAB-MiPs under the same transfection conditions resulted in higher levels of gene expression and even augmented the myogenic propensity (Supplemental Figure 6, D and E). Thus, the differential myogenic propensity of MAB-iPSCs and MAB-MiPs correlated with inherited biases in DNA methylation and $\mathrm{H} 3 \mathrm{~K}$ marks of key skeletal myogenesis marker genes. Also, altering expression levels of both mesodermal and skeletal myogenic genes in f-MiPs to levels comparable to those in MAB-MiPs correlated with increased myogenic propensity. In addition, the cardiomyogenic markers appeared not to be progeny biased and were marked by a permissive/open epigenetic state in MiPs.

Combined functional regeneration of striated muscles in dystrophic mice. We then addressed the relevance of MiP technology for in vivo simultaneous regeneration of both striated muscle types. We tested isogenic $\mathrm{GFP}^{+} \mathrm{f}$ - and MAB-MiPs for combined repair of cardiac and skeletal muscles in dystrophic cardiomyopathic $S g c b$-null mice (ref. 23, Figure 5A, and Supplemental Figure 7A). Four and eight weeks after parallel delivery of $5 \times 10^{5}$ cells to the myocardium and femoral arteries, the cardiac muscles appeared extensively and comparably engrafted by f- and MAB-MiPs, whereas the hind limb skeletal muscles showed greater engraftment of MAB-MiPs (Figure 5, B and C). Immunostaining-based quantitation of $\mathrm{GFP}^{+}$areas revealed that $\mathrm{f}$ - and MAB-MiPs engrafted $28.24 \% \pm 1.90 \%$ and $28.64 \% \pm 1.98 \%$ of the left ventricle wall (average $\pm \mathrm{SD} ; n=8$ mice/group; $P=0.23$ [NS], Mann-Whitney $U$ test), respectively. Conversely, quantitation of $\mathrm{GFP}^{+}$fibers in the gastrocnemius muscles revealed a significant difference in engraftment between f-MiPs $(9.30 \% \pm 0.74 \%)$ and MAB-MiPs $(31.33 \% \pm 2.20 \% ; P=0.0002)$. Consequently, we asked whether the engraftment paralleled the restoration of the missing protein. The reappearance of Sgcb was comparable in f-MiP- and MAB-MiP-engrafted hearts, whereas it appeared significantly increased in MAB-MiP-engrafted skeletal muscles (Figure 5D and Figure 6A). Likewise, fibrotic scars in both muscle types appeared to be comparably reduced (Supplemental Figure 7B). Furthermore, we sought to determine whether the molecular and histological features of MiP-driven regeneration matched the functional outcome. At 4 and 8 weeks after delivery, 3D echocardiography showed improved cardiac function, reduced hypertrophic remodeling, and decreased left ventricle dilation to a similar extent in f-MiP- and MAB-MiP-treated cohorts (Figure 6B and Supplemental Figure 7, $\mathrm{C}$ and D). However, performance on the treadmill was significantly better in MAB-MiP-treated mice compared with f-MiP-treated mice at both time points. In addition, creatine kinase (CK) serum levels were lower in MAB-MiP-injected mice than in f-MiP-injected mice under resting conditions. Also, 8 weeks after delivery, the curve of 


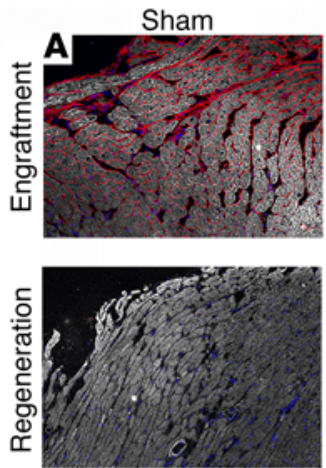

Myocd/Sgcb/G

$\mathrm{GFP}^{+}$cardiomyocytes $\mathrm{GFP}^{+} / \mathrm{Sgcb}^{+}$cardiomyocytes (\% of total)
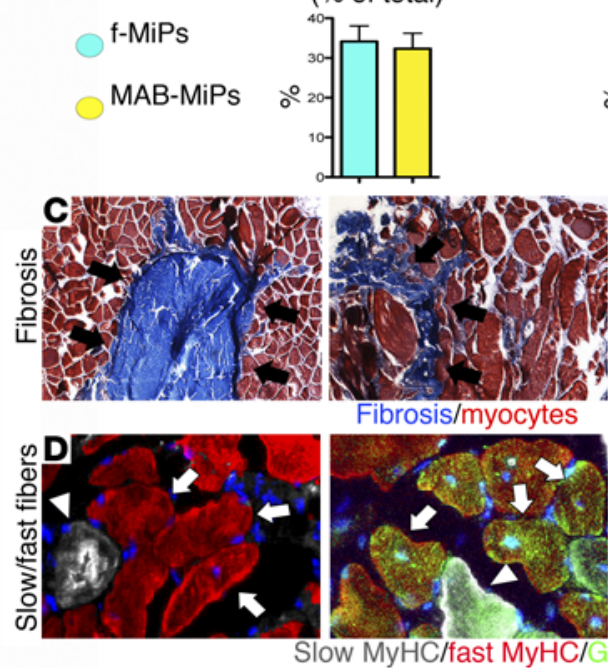

$\%$ of total fibers

$$
\% \text { of }
$$

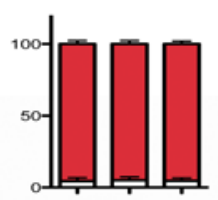

MAB-MiP
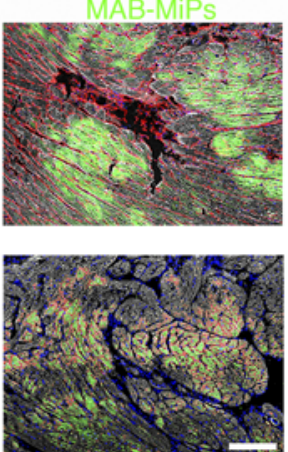

(\% of total)
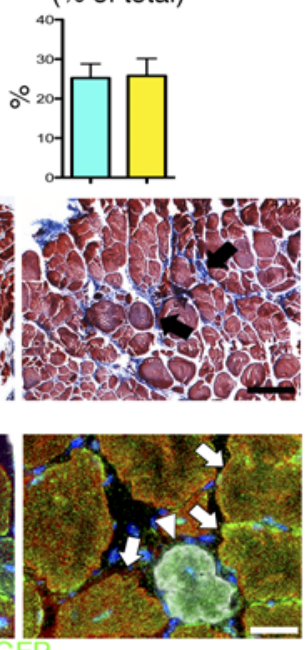

$\%$ of engrafted fibers

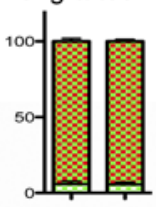

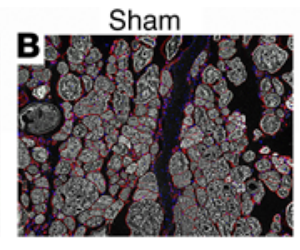
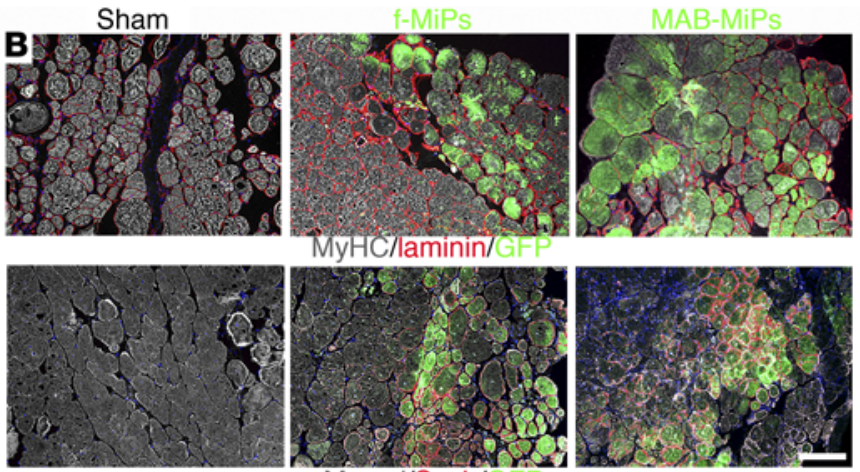

MyHCllaminin/ar
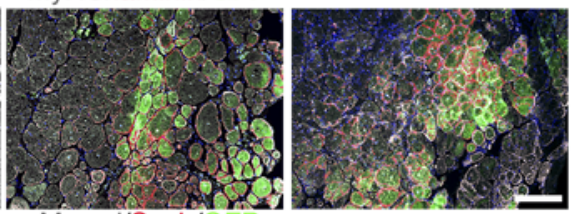

Myocd/Sgcb/

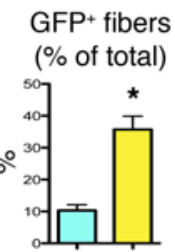

$\mathrm{GFP}^{+} / \mathrm{Sgcb}^{+}$fibers

(\% of total)
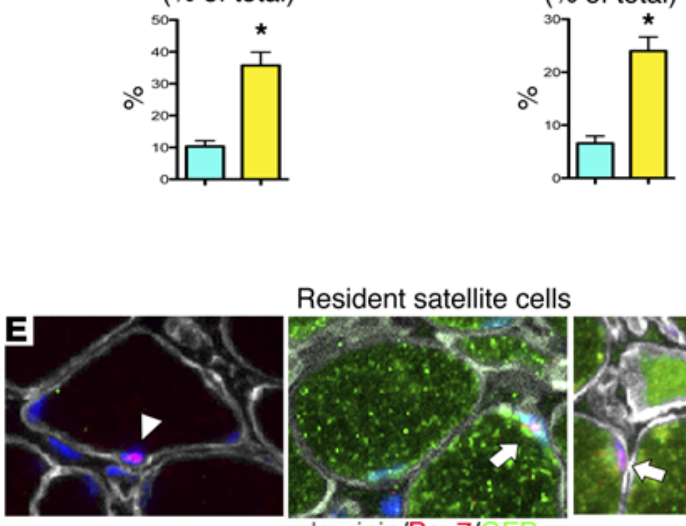

Resident satellite cells

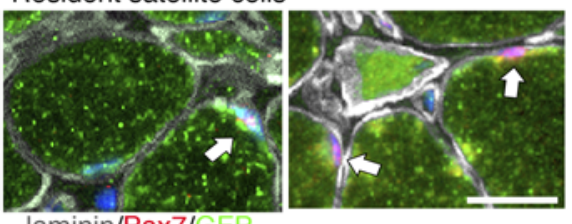

$\mathrm{GFP}^{+}$satellite cells (\% of total satellite cells)

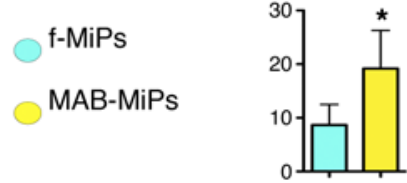

Figure 8. Single-cell MiP clones recapitulate the differential propensity for skeletal muscle regeneration. (A) Four weeks after injection, both $\mathrm{f}$ - and MAB-MiPs comparably engrafted the left ventricular wall, with no significant differences in GFP+ (engrafted) or GFP+Sgcb+ (regenerated) cardiomyocytes. (B) MAB-MiP clones engrafted and regenerated the hind limb muscles (shown are gastrocnemius muscles) to a significantly greater extent than did f-MiP clones, as quantitated in GFP+ and GFP+Sgcb fibers. $n=5$ mice/cohort. ${ }^{*} P<0.05$, Mann-Whitney $U$ test. (C) Masson's trichrome staining revealed conspicuous fibrotic scars (black arrows) in sham mice that were partially reduced in $\mathrm{f}$-MiP-injected mice and even more reduced in MAB-MiP-injected mice (shown are gastrocnemius muscles; $n=5$ mice/cohort). (D) Immunostaining for slow (arrowheads) and fast (arrows) fibers showed that both fiber types were engrafted in f-MiP- and MAB-MiP-injected mice (shown are gastrocnemius muscles). Quantitation of relative fiber type composition of whole-muscle sections and engrafted fibers revealed no significant differences among cohorts (data are related to gastrocnemius and tibialis anterior muscles; $n=5$ mice/cohort; Kruskal-Wallis and Mann-Whitney $U$ tests). (E) MAB-MiP clones engrafted the resident pool of Pax $7^{+}$satellite cells to a significantly greater extent than did f-MiPs. $n=5$ mice/cohort. ${ }^{*} P<0.05$, Mann-Whitney $U$ test. Error bars represent SD. Scale bars: $\sim 100 \mu \mathrm{m}(\mathbf{A}-\mathbf{C})$ and $50 \mu \mathrm{m}(\mathbf{D}$ and $\mathbf{E})$.

absolute force developed by extensor digitorum longus (EDL) muscles under iterated bouts of isometric contraction was increased to a significantly higher extent in MAB-MiP-injected mice than in f-MiP-injected mice when compared with that observed in sham controls (Figure 6C and Supplemental Figure 7D). Finally, we analyzed off-target engraftment of MiPs in filter organs. Eight weeks after delivery, qPCR analysis indicated barely detectable levels of GFP in the liver, lungs, spleen, and kidneys of MiP-injected mice as compared with levels in skeletal and cardiac muscles. Histological analysis of these organs showed a rare presence of $\mathrm{GFP}^{+}$small vessels (Figure 6D), suggesting very limited ectopic engraftment of injected MiPs in filter organs. In order to more stringently evaluate the myogenic propensity of f-MiPs versus that of MAB-MiPs in vivo, we obtained single-cell clones of GFP ${ }^{+}$MiPs (Figure 7A). qPCR analysis of mesodermal, skeletal, and cardiac myogenic markers in single MiP clones confirmed the expression patterns previously evaluated in whole-cell pools (Figure 7B). We then injected $S g c b$ null mice with single-cell MiP clones, following the same parallel delivery procedure. Four weeks after delivery, engraftment $\left(\mathrm{GFP}^{+}\right.$ myocytes) and regeneration (GFP $\mathrm{Sgcb}^{+}$myocytes) rates were comparable in cardiac muscles (Figure 8A), but these rates were significantly higher in the hind limb skeletal muscles of mice injected with 
A

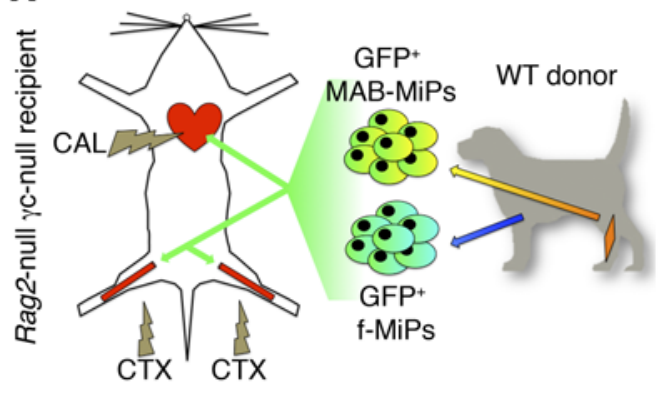

Sham
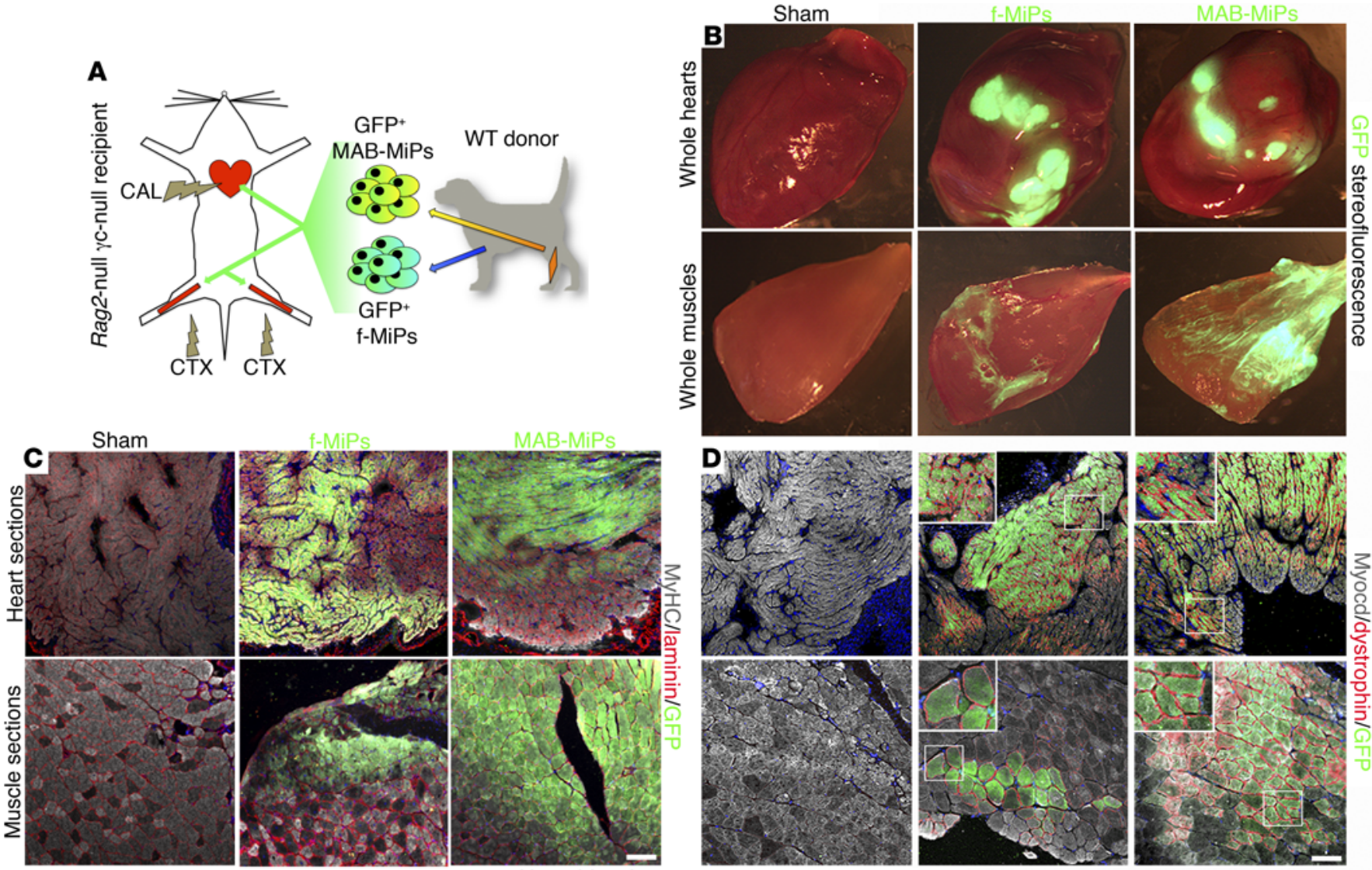

$\mathbf{E}$

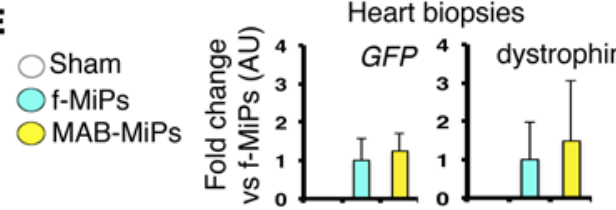

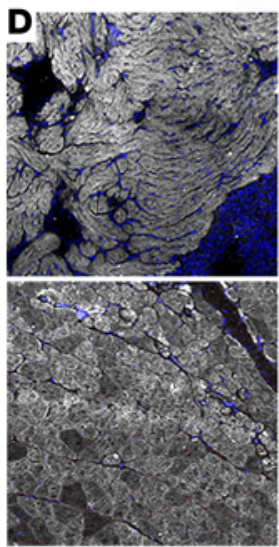
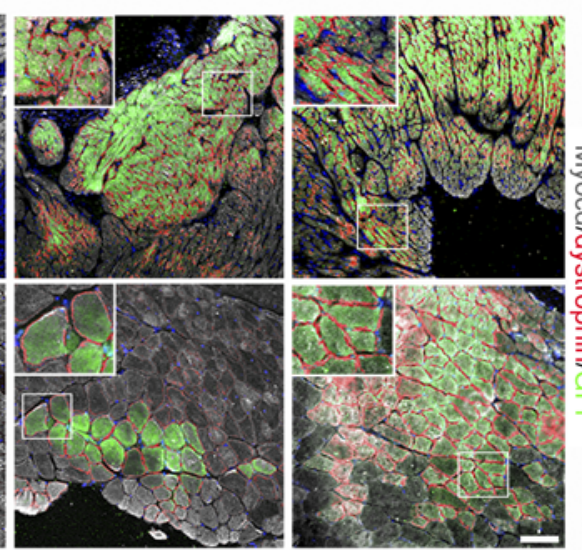

Muscle biopsies

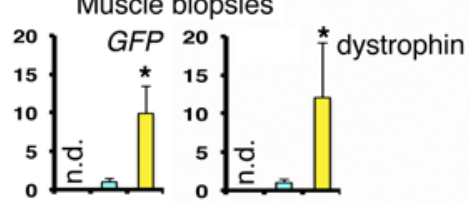

Figure 9. Translation of MiP-based in vivo approach in xenograft settings with canine cells. (A) Experimental plan of canine MiP dual injections in immunodeficient mice with heart damage induced by transient coronary artery ligation (CAL) and in the hind limb skeletal muscles induced by i.m. cardiotoxin (CTX) injections. Injected GFP+ $f$ - and MAB-MiPs were isolated from the same WT donor (number 2). Four weeks after injection, canine $f-$ and MAB-MiPs engrafted the myocardium at comparable levels, but not the hind limb muscles, where MAB-MiP engraftment rates appeared higher, as shown by stereofluorescence (B) (shown are tibialis anterior muscles) and immunostaining (C) (shown are gastrocnemius muscles). (D) Immunofluorescence analysis revealed comparable levels of GFP+ canine-specific dystrophin+ ${ }^{+}$cardiomyocytes in MiP-treated animals, but significantly greater numbers of GFP+dystrophin ${ }^{+}$myofibers in MAB-MiP-treated hind limb muscles. $n=7$ mice/group. (E) qPCR analyses consistently showed that $G F P$ expression levels were similar in injected hearts, yet increased in skeletal muscles (tibialis anterior), and that dystrophin levels followed a similar pattern. $n=7$ mice/group. ${ }^{* *} P<0.05$ versus $\mathrm{f}-\mathrm{MiPs}$, unpaired $t$ test. Error bars represent SD. Original magnification, $\times \mathbf{4 0}$ for insets of selected areas; scale bars: $100 \mu \mathrm{m}$.

MAB-MiP clones than in those of mice injected with f-MiP clones (Figure 8B). Fibrotic scars in the skeletal muscles appeared similarly reduced in MiP-injected hind limbs (Figure 8C). We then asked whether the progeny of MiP clones influenced engraftment toward a specific fiber type (slow or fast). Immunostaining analyses of slow and fast fibers in mixed muscles (i.e., gastrocnemius) revealed that both slow and fast fibers were engrafted by f- and MAB-MiPs. Also, we found no significant quantitative differences between sham and MiP-injected mice in the relative fiber type composition of whole muscles and engrafted fibers (Figure 8D). Finally, we assessed the contribution of MiP clones to the resident pool of $\mathrm{Pax}^{+}$satellite cells. Consistent with the engraftment and regeneration data, the $\mathrm{GFP}^{+} \mathrm{Pax} 7^{+}$fraction of resident satellite cells was significantly higher in MAB-MiP-treated muscles than in f-MiP-treated muscles (Figure $8 E)$. Thus, murine MiPs were able to simultaneously regenerate both striated muscle types after combined administration in vivo. Intriguingly, the myogenic propensity of MAB-MiPs did not influence cardiac muscle regeneration, but correlated with enhanced contribution to skeletal muscle repair.

Translation into canine settings. In order to gain translational insight into our system of combined striated muscle regeneration and skeletal muscle bias, we used the same MiP experimental protocol in a canine model. Golden retriever MD (GRMD) dogs still constitute the foremost preclinical large-animal model for novel MD regenerative treatments (24). In light of this fact, we isolated isogenic skin fibroblasts and skeletal muscle MABs from age-matched WT and GRMD dogs (Supplemental Figure 8, A and B), adapting the methods previously reported for human MABs (25). Given the strong similarity between canine reprogramming factors and human orthologs, we reprogrammed the 

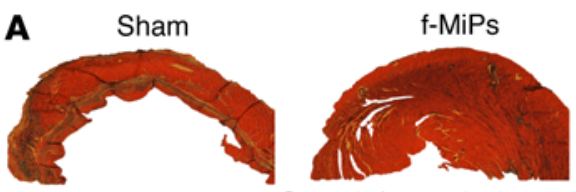

Outer left ventricular walls

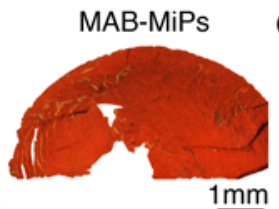

$1 \mathrm{~mm}$
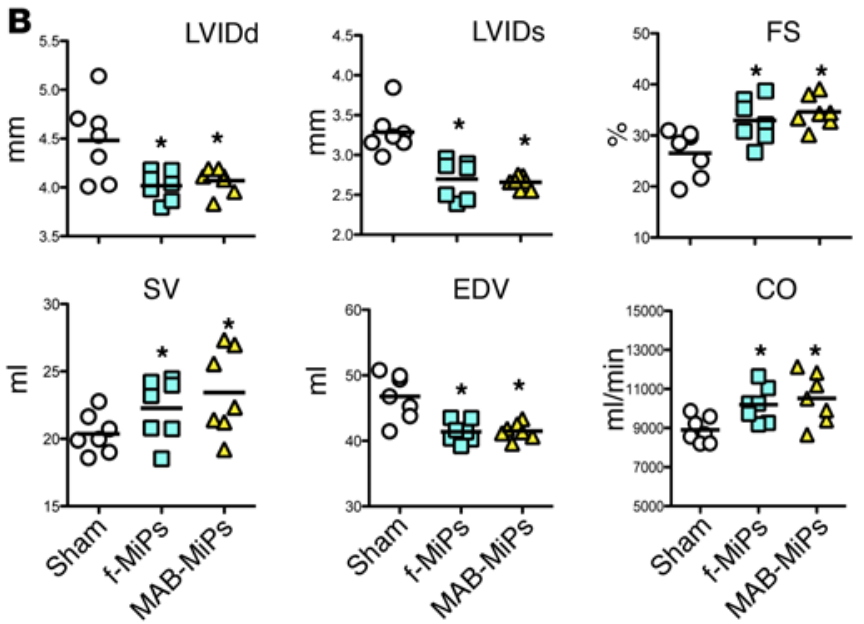
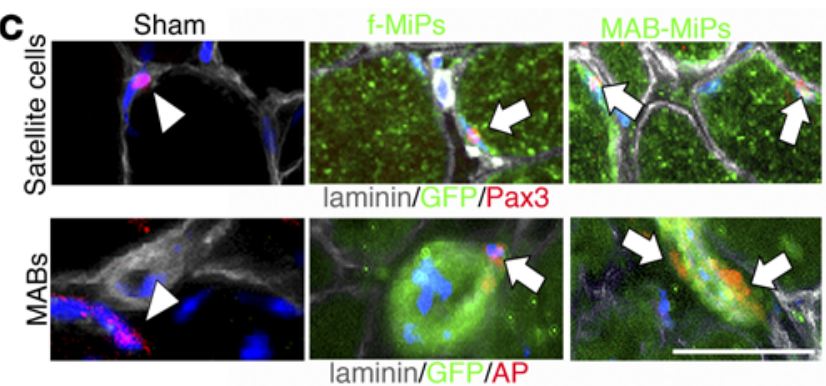

$\%$ GFP $+/ \mathrm{Pax}^{+}$cells vs $\mathrm{Pax}^{+}$cells/section

$\% \mathrm{GFP}^{+} / \mathrm{AP}^{+}$cells vs $\mathrm{AP}^{+}$cells/section

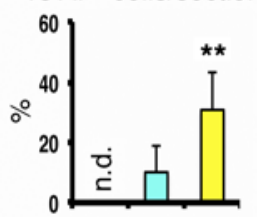

Figure 10. Canine MiPs regenerate the heart and engraft within resident stem cell pools in the skeletal muscle. (A) Masson's trichrome staining of whole-heart transversal sections showed dramatic amelioration of the outer left ventricular wall in terms of fibrosis and cardiomyocyte mass. (B) Accordingly, 3D echocardiographic results showed comparable improvement in adverse ischemic remodeling by $\mathrm{f}-$ and $\mathrm{MAB}-\mathrm{MiPs}$. $n=7 \mathrm{mice} / \mathrm{group}$. ${ }^{*} P<0.05$ versus sham, Kruskal-Wallis and Mann-Whitney $U$ tests. Each data point refers to 1 animal. Error bars represent average values. (C) Immunofluorescence imaging and quantitation showed that MAB-MiPs contributed in greater numbers to the quiescent pools of satellite cells (Pax $3^{+}$, underneath the sarcolemma) and $\mathrm{MABs}\left(\mathrm{AP}^{+}\right.$, juxtaposed with small vessels) in engrafted hind limb muscles than did $\mathrm{f}-\mathrm{MiPs}$ (arrowheads indicate GFP- resident cells; arrows indicate GFP+ engrafted resident cells). $n=7 /$ group. ${ }^{*} P<0.05$ versus f-MiPs, Mann-Whitney $U$ test. Scale bar: $\sim 50 \mu \mathrm{m}$.

canine cells with retroviral vectors carrying the human factors OCT4, SOX2, KLF4, and $C M Y C$. After individual clone selection and expansion, canine iPSCs exhibited human iPSC-like (hiPSC-like) morphology, homogeneous expression of endogenous pluripotency markers, and a normal karyotype (Supplemental Figure 8C). We then tested $\mathrm{GFP}^{+}$WT and GRMD iPSCs for skeletal muscle memory using the teratoma assay. Both WT and GRMD MAB-iPSCs showed greater differentiation toward skeletal muscle derivatives as compared with isogenic f-iPSC controls (Supplemental Figure 8D). Furthermore, we asked whether canine MiPs recapitulated the characteristics observed in murine MiPs. Canine MiPs appeared highly similar to murine MiPs with regard to morphology, molecular characteristics, and in vitro differentiation ability (Supplemental Figure 9, A-C). Consistent with our previous findings, canine MAB-MiPs showed a higher propensity toward skeletal muscle differentiation than did f-iPSCs in both WT and GRMD genotypes (Supplemental Figure 9D). Thus, the differential myogenic propensity was also identifiable in isogenic canine iPSCs from both WT and dystrophic animals and appeared durable in canine MAB-MiPs.

Assessment of canine MiP potential and GRMD correction. We then sought to investigate the in vivo regenerative potential of canine MiPs in an immunodeficient murine model (26) of parallel acute damage in both striated muscle types. Similarly to the murine MiP protocol, we administered isogenic WT canine $\mathrm{f}-$ and MAB-MiPs to age-matched Rag2-null $\gamma c$-null mice following myocardial injury induced by ischemia/reperfusion and hind limb skel- etal muscle injury induced by local cardiotoxin injection (Figure 9A). Four weeks after parallel delivery, canine f- and MAB-MiPs appeared comparably engrafted into the myocardium, whereas MAB-MiPs engrafted into skeletal fibers with higher efficiency than did f-MiPs (Figure 9, B and C). Immunostaining-based quantitation of $\mathrm{GFP}^{+}$areas revealed that $\mathrm{f}-$ and MAB-MiPs engrafted $28.82 \% \pm 1.39 \%$ and $27.68 \% \pm 2.44 \%$ of the left ventricular wall ( $n=7$ mice/group; $P=0.71$ [NS], Mann-Whitney $U$ test). Conversely, quantitation of $\mathrm{GFP}^{+}$fibers in the gastrocnemius muscles revealed a significant engraftment difference between f-MiPs $(10.59 \% \pm$ $7.71 \%)$ and MAB-MiPs $(33.98 \% \pm 1.83 \% ; P=0.0006)$. We thus asked whether the engraftment paralleled the regeneration, evaluated as expression of nonmurine dystrophin. Accordingly, MiPinjected tissues showed comparable levels of dystrophin in engrafted ventricles and higher levels in MAB-MiP-treated versus f-MiP-treated skeletal muscles (Figure 9, D and E). Interestingly, f- and MAB-MiPs comparably counteracted adverse ischemic remodeling (Figure 10, A and B, and Supplemental Figure 10A), whereas MAB-MiPs contributed at significantly greater numbers of cells to the resident progenitor pools (satellite cells and MABs) in treated skeletal muscles as compared with f-MiPs (Figure 10C). Subsequently, we sought to conjugate the MAB-MiP-based approach with genome editing and mutation correction. We used tailored TALENs and donor matrix plasmids (27) to specifically correct the GRMD mutation in canine dystrophic MAB-iPSCs (Supplemental Figure 10B). After single-cell dilution and targeting validation, corrected MAB-iPSCs were also checked for pluripo- 
A

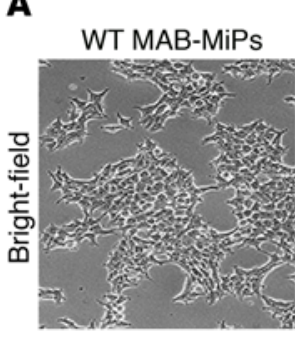

B

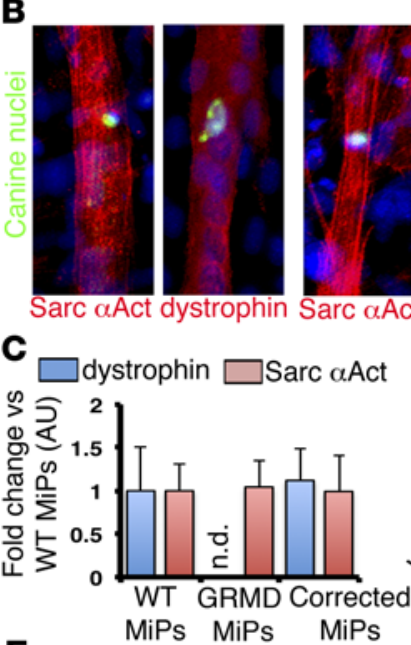

E

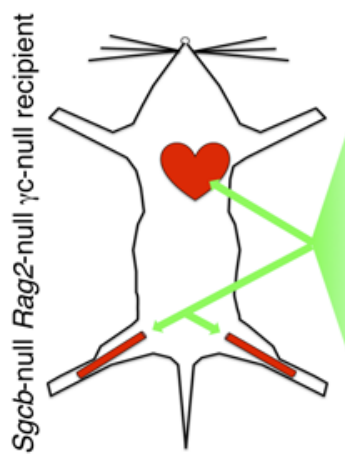

TALEN-corrected

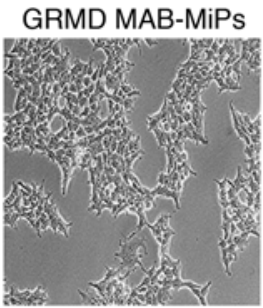
GRMD MAB-MiPs
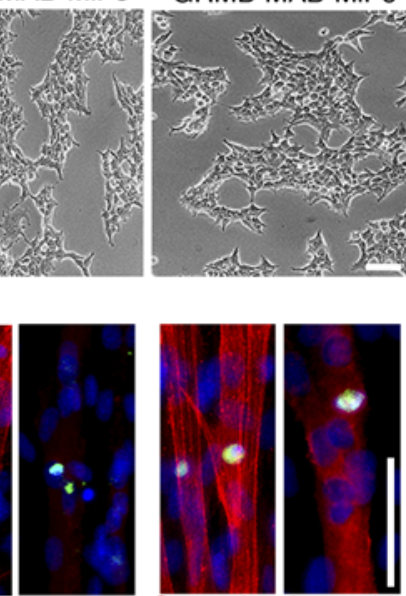

dystrophin Sarc $\alpha$ Act dystrophin

D

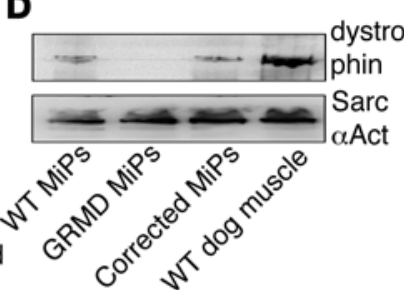

GFP+
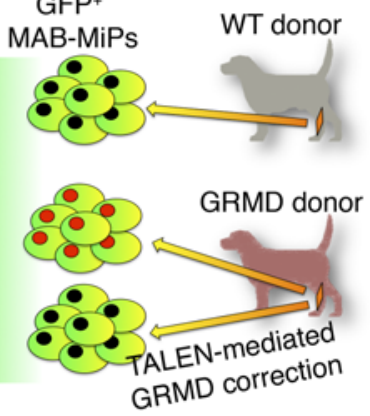

$\mathbf{F}$
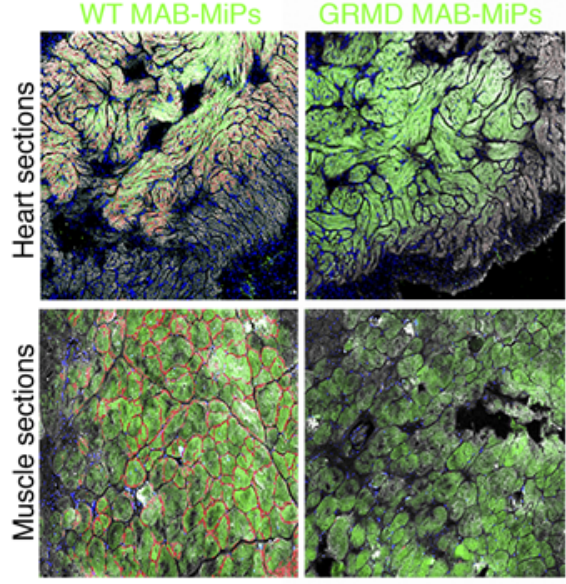

G Heart extracts
dystro-
phin
GFP

$\mathrm{MyHC}$

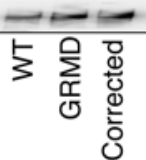

H
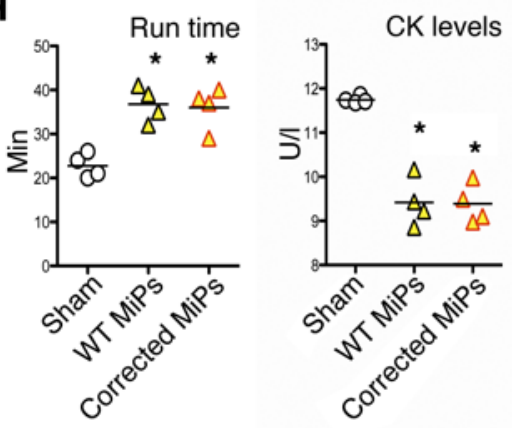
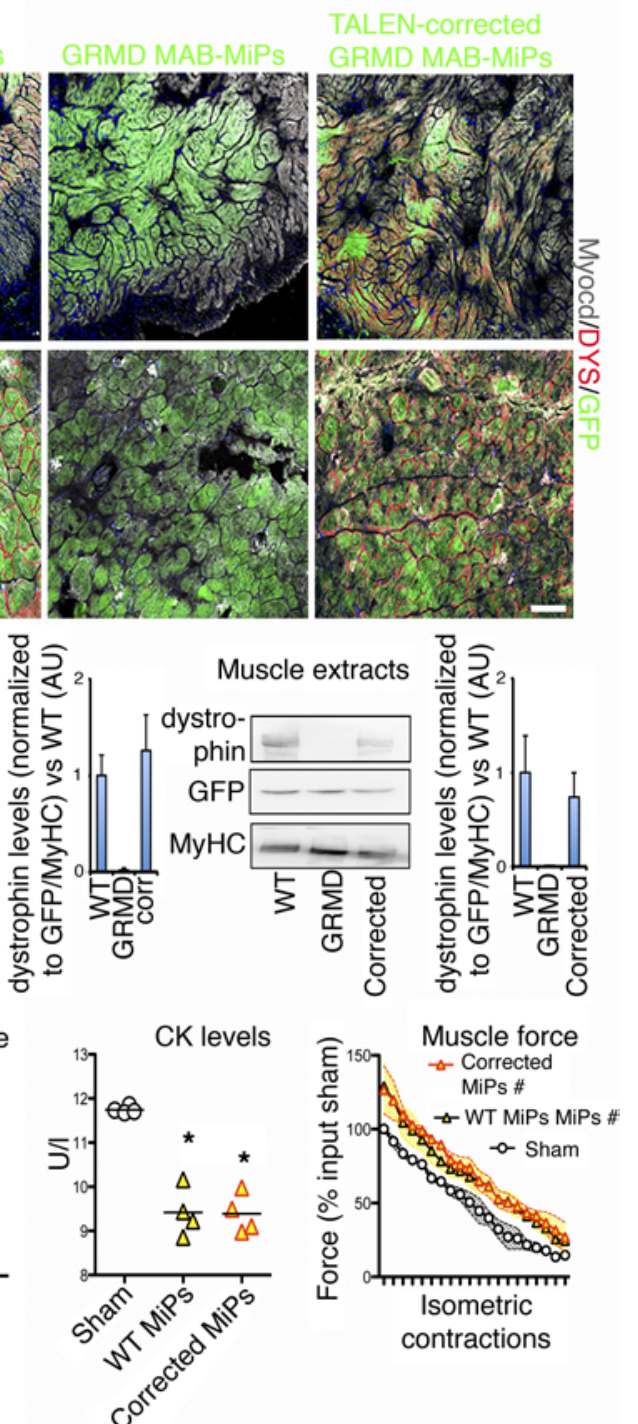

Figure 11. TALEN-mediated correction of GRMD mutation and application in vivo. Proliferation of WT, GRMD, and TALEN-corrected MAB-MiPs was indistinguishable (A), yet canine-specific dystrophin was detected only when WT and corrected MiPs were differentiated in low-ratio coculture with murine myoblasts, as shown by immunofluorescence (B), qPCR (C), and WB (D) analyses. (E) Schematic representation of the in vivo proof of TALEN-based canine MiP correction in dystrophic immunodeficient mice. Four weeks after injection, all MAB-MiPs robustly engrafted cardiac and skeletal muscles of $S g c b-n u l l$ Rag2-null $\gamma$ c-null mice, but dystrophin was detected at comparable levels only in WT and corrected MiP-engrafted tissues, as shown by immunofluorescence imaging $(\mathbf{F})$ and WB and densitometric analyses $(\mathbf{G})$, albeit with interindividual variability. $n=4$ mice/group. Kruskal-Wallis and Mann-Whitney $U$ tests. (H) Four weeks after in vivo delivery, WT and corrected MAB-MiPs comparably induced improved treadmill performance, decreased serum CK levels, and increased the absolute force curve upon iterated isometric contractions. $n=4$ mice/cohort. ${ }^{*} P<0.05$ versus sham, Kruskal-Wallis and Mann-Whitney $U$ tests. Each data point represents 1 animal. $n=4$ mice/cohort. ${ }^{P} P<0.05$ versus sham, 2 -way ANOVA. Data represent average values, expressed as a percentage of input sham (average value at approximately 1 for sham muscles); ribbons depict the interval of SD. Scale bars: $100 \mu \mathrm{m}$.

tency marker expression and normal karyotype (Supplemental Figure 10C). Once isolated and cocultured with myoblasts, corrected GRMD MAB-MiPs efficiently rescued dystrophin expression as compared with that observed in WT and uncorrected controls (Figure 11, A-D). To prove TALEN-based MiP correction of chronic muscle wastage in vivo, we obtained a suitable dystrophic, xenograft-permissive model. We crossed Sgcb-null and Rag2-null $\gamma c$-null mice and validated the dystrophic degeneration in $S g c b$-null Rag2-null $\gamma c$-null striated muscles (Supplemental Figure 11A). We then tested $\mathrm{GFP}^{+} \mathrm{WT}$, GRMD, and isogenic corrected MAB-MiPs via parallel delivery into $S g c b$-null Rag2-null $\gamma c$-null mice (Figure 11E). Four weeks after injection, dystrophin was detected at comparable levels in WT- and corrected MiP-engrafted cardiac and skeletal muscles, albeit with interindividual variability (Figure 11, $\mathrm{F}$ and $\mathrm{G}$ ). In addition, treadmill performance, serum CK levels under resting conditions, and absolute force in response to iterated bouts of isometric contraction were ameliorated to a comparable extent in mice injected with WT or corrected MiPs as compared with sham-injected mice (Figure 11H). Thus, the MiP-based dual treatment of striated muscles appeared translatable to xenograft, 
A

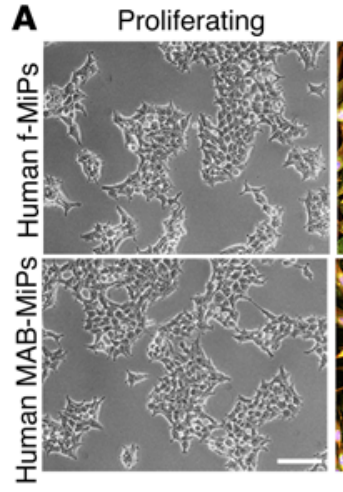

Cardiac muscle

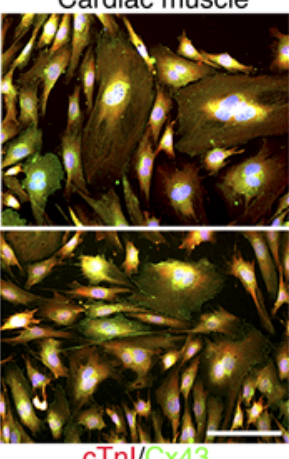

Skeletal muscle

$\% \mathrm{cTnl}^{+}-\mathrm{Cx} 43^{+}$ cells/total cells

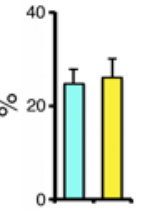

f-MiPs

MAB-MiPs

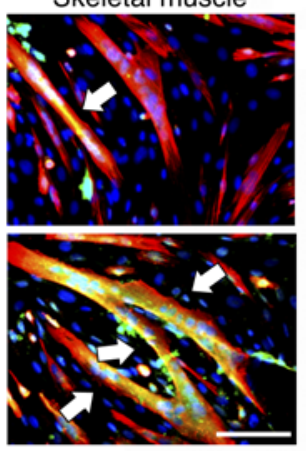

$\% \mathrm{GFP}^{+}$myotubes/

total myotubes

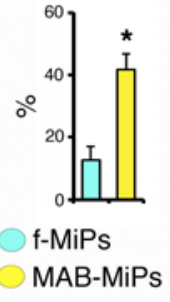

B Osteogenic

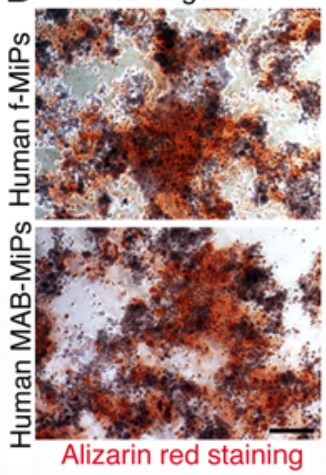

Adipogenic

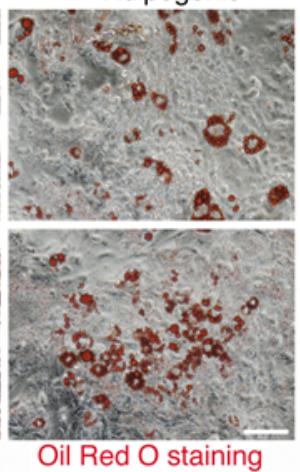

Endothelial

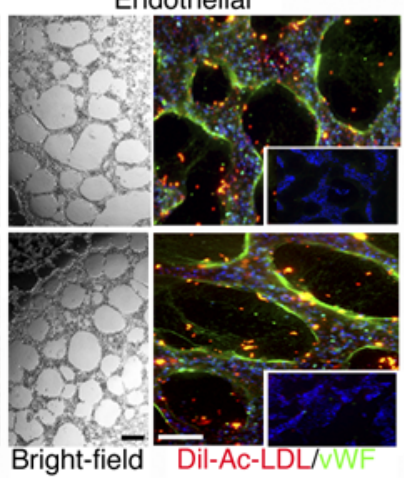

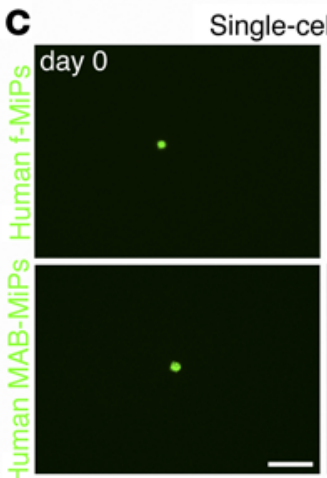
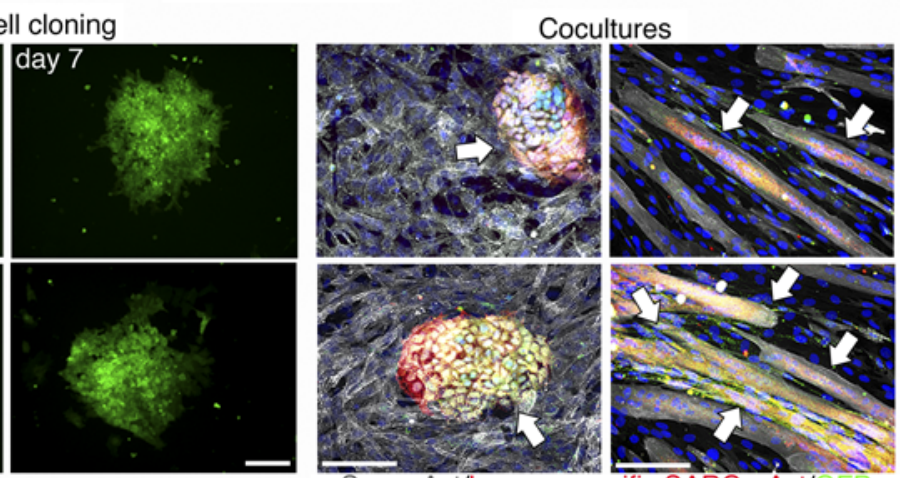

Sarc aAct/human-specific SARC $\alpha$ Act/GF

Figure 12. In vitro characterization of myogenic propensity in human MiPs. (A) After triple sequential sorting for CD140a/CD140b/CD44 from iPSC clones obtained from 3 independent donors, isogenic human $\mathrm{GFP}^{+} \mathrm{f}-$ and MAB-MiPs displayed similar morphology. Consistent with murine and canine cells, $\mathrm{f}-$ and MAB-MiPs showed comparable efficiency in their differentiation toward cardiomyocyte-like (cTnl+- $\mathrm{Cx} 43^{+}$) cells, whereas human MABMiPs showed greater myogenic potential in coculture with C2C12, evaluated as a propensity toward GFP+ chimeric myotubes (arrows). $n=4 / \mathrm{MiP}$ type. ${ }^{*} P<0.05$ versus $f-M i P s$, Mann Whitney $U$ test. Error bars represent SD. (B) Differentiation toward osteogenic, adipogenic, and endothelial lineages was robust and comparable between human f- and MAB-MiPs. $n=4 /$ MiP type. Insets in B show negative staining control and proliferating cells at equal magnification (original magnification, $\times 20$ ). (C) After single-cell clonal expansion, f-MiP and MAB-MiP clones were cocultured with cardiomyocytes and myoblasts of rodent origin. Immunostaining for human-specific Sarc $\alpha$ Act showed that the human-specific sarcomeric protein specifically colocalized with GFP+ engrafted cardiomyocyte clusters and myotubes (arrows), thus supporting the notion that MiP fusion correlates with terminal differentiation. $n=3 /$ MiP type. Scale bars: $\sim 100 \mu \mathrm{m}$.

canine-in-murine settings. Also, the MAB-related myogenic bias influenced skeletal myogenic, but not cardiomyogenic, regeneration of canine MiPs. Finally, the MiP-based approach proved suitable for targeted MD correction in vivo.

Proof of principle using isogenic human cells. Finally, as proof of principle in human settings, we isolated isogenic fibroblasts and MABs from fetal biopsies of skin and muscle tissues, respectively (Supplemental Figure 11B). After reprogramming with retroviral vectors, human $\mathrm{f}$ - and MAB-iPSCs showed comparable morphol- ogy and patterns of pluripotency marker expression (Supplemental Figure 11C). Consistent with murine and canine iPSCs, human MAB-iPSCs also spontaneously showed a higher propensity toward the skeletal muscle lineage compared with isogenic f-iPSCs, as observed in teratomas (Supplemental Figure 11D) and during in vitro differentiation after pulsed expression of PAX7 and MEF2C (Supplemental Figure 11E). Finally, once derived from these iPSCs, human isogenic $\mathrm{f}$ - and MAB-MiPs showed comparable levels of cardiomyogenic differentiation toward immature cardiomyocyte- 
like cells. However, MAB-MiPs showed skewed levels of skeletal muscle commitment in vitro (Figure 12A). In addition, human fand MAB-MiPs differentiated to a comparable extent toward osteogenic, adipogenic, and endothelial cell lineages, thus recapitulating the characteristics of murine and canine MiPs (Figure 12B). Finally, we used human MiPs to specifically address the question of whether, after MiPs fuse in vitro and in vivo, the presence of GFP in cardiac and skeletal myocytes is the result of unspecific GFP diffusion, or whether it correlates with specific lineage commitment. To this end, we obtained single-cell clones of human $\mathrm{GFP}^{+} \mathrm{f}-$ and MAB-MiPs and differentiated them in vitro with neonatal cardiomyocytes and myoblasts, both from rodents. Immunostaining with human-specific Abs revealed that only when $\mathrm{GFP}^{+}$ MiPs fused with cardiomyocyte clusters or skeletal myotubes was the signal of human sarcomeric proteins detectable, and it largely colocalized with the GFP signal (Figure 12C), indicating specific myogenic commitment upon GFP diffusion. Thus, MAB-related enhanced myogenic propensity appeared evident and durable also in human iPSCs and MiPs. Moreover, human MiPs recapitulated, at least in vitro, the capability of dual differentiation toward the striated muscle lineages, with an enhanced propensity for skeletal myogenesis in human MAB-MiPs.

\section{Discussion}

Multitissue differentiation potential is one of the most appealing aspects of iPSC technology for MD treatments, in which combined rescue of cardiac and skeletal muscle functionality is paramount (28). Yet the combination of safety and efficaciousness of iPSC-derived cells in vivo remains an important issue for the translation of these methods from bench to bedside (29). In this view, a refined understanding of the source-related retainment of myogenic propensity and its in vivo relevance may provide a relatively easy way to tune the intrinsic fate of iPSCs and derived progenitors by means of donor cell choice. Our results indicate that the enhanced myogenic propensity of MAB-derived iPSCs and MiPs correlates with the retained epigenetic cues of histone marks and DNA methylation of $\mathrm{CpG}$ islands upstream of key markers of lineage progression. Indeed, in our experimental settings, the permissive/activating epigenetic cues found on cardiomyogenic markers correlated with a robust cardiomyogenic potential of MiPs, especially as shown in vivo. Conversely, progeny-related retainment of different epigenetic signatures on skeletal muscle genes was associated with a differential intrinsic propensity of MiPs toward the skeletal muscle lineage. We are aware that our epigenetic analyses were limited to a small number of regulatory elements and genes and are thus only sufficient for describing a correlation between the differential propensity of $\mathrm{f}$ - and MABderived cells. In order to gain mechanistic insights into how the epigenetic imprinting is retained and causes the skew in intrinsic propensity, a more comprehensive, genome-wide approach will be needed. Importantly, such an approach will likely identify putative key regulators of myogenic bias retainment and, thereby, putative targets to specifically increase or decrease skeletal myogenic propensity during reprogramming and differentiation.

In our study, we used MAB-derived iPSCs and MiPs as sources of myogenic bias in the context of striated muscle regeneration and fibroblast-derived cells as controls. We chose this cell model because its myogenic bias has already been partially documented (11), and its intrinsic propensity was hypothesized to be of potential relevance in the context of muscle repair. For a starting somatic cell population, we consistently isolated MABs as an alkaline phosphatase-sorted (AP-sorted) subfraction of pericytes in all three species. Although the mechanisms are still largely unknown, AP apparently marks a myogenic subset of resident pericytes in the skeletal muscle that are locally committed to skeletal myogenesis, especially in vivo (30). Importantly, the intrinsic myogenic potential of resident and injected murine MABs for differentiation into skeletal muscle tissue has been confirmed in a transgenic, inducible genetic system relying on AP::CreERT2 recombinase (31). Also, although restricted to MABs of embryonic origin, genetic ablation of Pax3 blunts the skeletal myogenic potential of MABs (32). Moreover, by means of a dedicated scaffold, murine MABs were shown to generate functional artificial skeletal muscle tissue in vivo (33). Thus, resident $\mathrm{AP}^{+} \mathrm{MABs}$ from skeletal muscles appear intrinsically myogenic, probably to a species-specific variable extent, and, as shown by the present work, this intrinsic myogenic bias appears durable after reprogramming in murine, canine, and human settings. We did not use satellite cells as a source for iPSCs, as it was already reported that committed myoblasts are extremely inefficient to reprogram and that less committed, multipotent resident stem cells from the skeletal muscle were more efficiently reprogrammable (34). Furthermore, albeit demonstrated in an unrelated lineage, i.e., pancreatic adenocarcinoma, it has been shown that iPSCs generated from undifferentiated cells (tumor cells) produced more mature, lineage-specific derivatives when compared with iPSCs generated from differentiated cells (margin cells), thus indicating the possibility of efficient propensity retainment from less committed cells (35). Besides the differences in myogenic commitment, we did not observe significant shifts in endothelial differentiation of $\mathrm{f}$ - versus MAB-MiPs either in vitro or in vivo. However, in murine muscles injected with canine cells, higher rates of MAB-MiP engraftment and regeneration in skeletal muscle correlated with higher rates of engraftment in the pool of resident $\mathrm{AP}^{+} \mathrm{MABs}$. More comprehensive studies are thus required to shed light on the molecular cues that possibly discriminate between pericytic and myogenic propensities in these cells. In this perspective, an intriguing experimental question would be whether reprogrammed MABs retain a similar propensity, such as a that toward other mesodermal lineages (e.g., endothelial, osteogenic, adipogenic), when generated from somatic MABs exposed to other differentiative cues. However, such an approach will necessarily face the potential restriction in general properties of transdifferentiated MABs, such as proliferation and susceptibility to reprogramming, which would influence the quality of iPSCs and possibly mask the changes in intrinsic propensity.

Several reports have recently pointed to the transient nature of epigenetic memory and its washout after continuous passaging (8). We cannot exclude the possibility that this could also be a limitation in our system, since our experiments of morula aggregation, teratoma formation, and $\mathrm{MiP}$ isolation were conducted with iPSCs at low $(\leq 5)$ passage numbers. However, passaging-induced fadeout of epigenetic imprinting could, in principle, be compensated by the tremendous expansion capacity of both iPSCs and MiPs. In fact, under opportune conditions of reprogramming and expansion 
in a limited number of passages, myogenic imprinting could prove durable up to the MiP stage, even under good laboratory practice/ good manufacturing practice (GLP/GMP) conditions. Moreover, of importance for putative future therapies is the notion that epigenetic imprinting appears intrinsically more durable in human cells than in rodent cells (15). However, it is important to consider that epigenetic memory is not specifically tunable as such, at least not without the help of transgenic or genome-edited systems. Also, epigenetic bias is likely to vary in terms of imprinted genes according to the intrinsic status of the source cell and, therefore, to the cell preparation. Thus, it will be relevant for the field to use such skewed cellular systems to identify putative imprinted targets that affect cell potency. Nonintegrative manipulation of such candidates might then offer a more clinically oriented perspective for these studies.

The positive outcome we observed in treated dystrophic mice suggests that MiPs represent a promising tool for combined regeneration of both striated muscle types. In vitro and in vivo, MiPs were able to differentiate toward both cardiac and skeletal muscle lineages. Skeletal muscle differentiation of MiPs, particularly of murine origin, seemed to correlate with expression levels of mesodermal transition markers, e.g., brachyury, and of lineage-specific genes, e.g., Pax7. Despite the fact that no significant differences were found in brachyury expression when we analyzed the differentiating iPSC bulk cultures, isolated MAB-MiPs showed significantly higher expression levels of this gene as well as of Pax7 when compared with f-MiPs in both whole-cell pools and singlecell clones. Intriguingly, restoring the expression levels of both genes - but not each singularly - in f-MiPs markedly increased skeletal muscle differentiation in vitro to levels comparable to those observed with MAB-MiPs. Further studies are needed to determine whether such manipulation might also prove useful in in vivo settings. With regard to the spontaneous cardiac differentiation of MiPs, their high efficiency in generating cardiac-like cells in vitro was counterbalanced by their immature state (absence of spontaneous beating, immature pattern of terminal markers). Further studies are thus required to augment the terminal maturation of MiP-derived myocytes in vitro. However, the coculture systems we used showed functional maturation of the MiPs toward both striated muscle lineages, e.g., beating cardiomyocytes and dystrophin-expressing myotubes. Albeit more heterogeneous, the coculture systems enabled us to address the question of whether MiPs are competent for regeneration in the presence of surrounding myocytes, thus mimicking in vivo engraftment. Consistent with this notion, both murine and canine MiPs were indeed able to drive the combined functional regeneration of both striated muscle types in vivo in murine models of simultaneous delivery. Comparing the in vitro coculture and the robust rates of in vivo engraftment, we can speculate that MiPs mainly regenerate the striated muscles in vivo by fusing with resident myocytes and then differentiating into myocytes and regenerating the host muscle, as proven by reexpression of the missing therapeutic proteins (Sgcb, dystrophin), colocalization of GFP and MiP-specific sarcomeric proteins (human sarcomeric $\alpha$-actinin [Sarc $\alpha$ Act]), and the improved functional performance. In this regard, the levels of MiP-induced functional repair in dystrophic or injured mice were lower than WT levels in comparable experimental conditions (e.g., fractional shortening, $\sim 42 \%$; run time, $\sim 110 \mathrm{~min})$. However, the functional improvement was coherent across many parameters for both cardiac and skeletal muscles. This successfully addresses the main challenge facing the MiPs, namely, whether these novel progenitors are able to regenerate both striated muscle types with a combined, balanced outcome. In addition, both $\mathrm{f}$ - and MAB-MiPs appeared able to engraft both slow and fast muscle fibers and did not induce a quantitative shift in fiber type composition in engrafted muscles. Also, the off-target engraftment of MiPs after intracirculatory delivery appeared rare and restricted to vascular smooth muscle. Nevertheless, a better strategy to more efficiently infuse both types of striated muscle tissues with MiPs will likely increase the chances of greater functional restoration. Also, a deeper understanding of MiP biology will enable us to appropriately engineer MiPs for improved regenerative outcomes. Furthermore, the safety and efficacy of the MiP strategy will likely necessitate validation in large MD animal models in order to test its feasibility in human-sized striated muscles. In this view and with the goal of upgrading in vivo administration from targeted to systemic delivery, it will be of fundamental importance to identify and manipulate pathways that regulate MiP migration and homing.

Finally, the successful combination of MD mutation correction and in vivo regeneration of striatal muscle in immunodeficient dystrophic mice paves the way for further exploration of MiP-based approaches for putative autologous treatments. In this perspective, it was intriguing to observe that human MiPs recapitulated the dual myogenic commitment, even though our proof of principle was restricted to in vitro assessment. Therefore, a refined assessment of the in vivo regenerative potential of human MiPs will be crucial for progression along the translational path of MiP-based therapies for striated muscle regeneration.

\section{Methods}

Cell culture and isolation. Murine fibroblasts and MABs were isolated from 3 syngeneic C57/Bl6 male mice (obtained from internal stock at the KU Leuven Animal Facility). Murine fibroblasts were isolated by digesting minced tail tips in $0.1 \%$ collagenase IV (Thermo Fisher Scientific) in three 20 -minute rounds of shaking at $37^{\circ} \mathrm{C}$. Cells were single-cell cloned and expanded on gelatin-coated vessels in DMEM $20 \%$ medium (high-glucose DMEM supplemented with 20\% FBS, $1 \%$ sodium pyruvate, $1 \%$ penicillin/streptomycin, $1 \%$ L-glutamine, $1 \%$ nonessential amino acids [NEAA], and 0.2\% 2-mercaptoethanol) (Thermo Fisher Scientific) in $5 \% \mathrm{O}_{2} / 5 \% \mathrm{CO}_{2}$ at $37^{\circ} \mathrm{C}$ and checked for homogeneous expression of CD90. Murine MABs were isolated as previously described (25). Briefly, tibialis anterior muscles were tissue cultured in small pieces on collagen-coated $3.5-\mathrm{cm}$ dishes (Nunc, Thermo Fisher Scientific) in DMEM 20\% medium supplemented with $5 \%$ penicillin/streptomycin and $0.5 \%$ gentamycin (Sigma-Aldrich). Cells propagating from the biopsies were then FACS sorted as an $\mathrm{AP}^{+}$ fraction using a PE-conjugated Ab (R\&D Systems; catalog FAB1448P). $\mathrm{AP}^{+} \mathrm{MABs}$ were single-cell cloned, then expanded and checked for homogeneous AP expression before passage number 3 by means of 5-bromo-4-chloro-3-indolyl phosphate (BCIP) staining (SigmaAldrich) and/or live staining (Thermo Fisher Scientific). MAB clones were expanded on collagen-coated vessels (Nunc, Thermo Fisher Scientific) in DMEM $20 \%$ medium in $5 \% \mathrm{O}_{2} / 5 \% \mathrm{CO}_{2}$ at $37^{\circ} \mathrm{C}$.

Murine cells were reprogrammed as previously described (11). Briefly, cells were transduced with retroviral particles carrying Oct4, 
Sox2, Klf4, and cMyc cDNA sequences (pMX vectors; Addgene) and seeded at low confluence on a feeder layer of mitomycin-treated primary murine embryonic fibroblasts (iMEFs). Embryonic stem cell-like (ESClike) colonies were manually picked 3 to 4 weeks after transduction, clonally expanded, and characterized for pluripotency marker expression.

Murine $f-$ and MAB-iPSCs were cultured on a feeder layer of iMEFs in iPSC medium consisting of high-glucose DMEM supplemented with $20 \%$ knockout serum, $1 \%$ sodium pyruvate, $1 \%$ penicillin/streptomycin, 1\% L-glutamine, 1\% NEAA, 0.2\% 2-mercaptoethanol, and $100 \mathrm{U} /$ $\mathrm{ml}$ leukemia inhibitory factor (LIF) (all from Thermo Fisher Scientific except LIF, which was purchased from EMD Millipore) in $5 \% \mathrm{O}_{2} / 5 \%$ $\mathrm{CO}_{2}$ at $37^{\circ} \mathrm{C}$. $\mathrm{GFP}^{+}$iPSCs were obtained by transfecting the cells with a piggyBac transposon-bearing GFP and a dedicated transposaseexpressing plasmid, provided by M. Chuah and T. Vandendriessche (Department of Gene Therapy and Regenerative Medicine, VUB University, Brussels, Belgium). $\mathrm{GFP}^{+}$cells were then isolated by FACS 10 days after transfection. Once the feeder layer was removed by brief preplating on gelatin-coated plastic vessels (Nunc, Thermo Fisher Scientific), $\mathrm{GFP}^{+}$iPSCs were induced to form embryoid bodies (EBs) in suspension culture in nonculture-treated plastic dishes $\left(10^{5}\right.$ cells in an 8-ml/10-cm dish; BD) in DMEM $20 \%$ medium for 72 hours in $5 \% \mathrm{O}_{2} / 5 \%$ $\mathrm{CO}_{2}$ at $37^{\circ} \mathrm{C}$. Subsequently, EBs were collected, centrifuged, disaggregated by thorough pipetting, resuspended as single cells and seeded for suspension culture in the same number of nonculture-treated dishes used in the previous step, and placed in EB medium containing Iscove's modified Dulbecco's medium (IMDM) supplemented with 15\% FBS, $1 \%$ penicillin/streptomycin, 1\% L-glutamine (all from Thermo Fisher Scientific), $4.5 \mathrm{mM}$ thyoglycerol, $50 \mathrm{mg} / \mathrm{ml}$ ascorbic acid, and 200 $\mathrm{mg} / \mathrm{ml}$ holo-transferrin (all from Sigma-Aldrich) for 72 hours in $5 \%$ $\mathrm{O}_{2} / 5 \% \mathrm{CO}_{2}$ at $37^{\circ} \mathrm{C}$. Next, EBs were gently collected, with care taken to avoid disruption, and plated on an equal number of collagen-coated, culture-treated plastic dishes (Nunc, Thermo Fisher Scientific) in EB medium for 72 hours in $5 \% \mathrm{O}_{2} / 5 \% \mathrm{CO}_{2}$ at $37^{\circ} \mathrm{C}$. At this stage, cells were gently detached with TrypLE (Thermo Fisher Scientific) and FACS sorted on a BD FACSAria III using APC-conjugated anti-CD140a Ab (eBioscience). Both CD140a ${ }^{+}$and CD140a- fractions were collected for further positive and negative selections, respectively, and seeded in DMEM $20 \%$ medium on collagen-coated $3.5-\mathrm{cm}$ dishes and incubated in $5 \% \mathrm{O}_{2} / 5 \% \mathrm{CO}_{2}$ at $37^{\circ} \mathrm{C}$. Upon $90 \%$ confluence, in conditions similar to those of the first sorting step, cells were further purified using APC-conjugated CD140b Ab, and, as a last step, CD 44 Ab (eBioscience; catalogs 17-1401-81, 17-1402-80, and 17-0441-81). After the three sorting steps, $\mathrm{CD}_{140} \mathrm{CD}^{+} 14 \mathrm{Ob}^{+} \mathrm{CD} 44^{+}$cells (murine MiPs) were cultured on collagen-coated, culture-treated plastic vessels (Nunc, Thermo Fisher Scientific) in DMEM $20 \%$ medium in $5 \% \mathrm{O}_{2} / 5 \% \mathrm{CO}_{2}$ at $37^{\circ} \mathrm{C}$. Single-cell cloning was performed by seeding murine MiPs at a density of 0.5 cells per well in flat-bottomed, collagen-coated 96-multiwell plates (Nunc, Thermo Fisher Scientific). After 9 to 10 days, proliferating clones were then expanded on 6-well plates for qPCR-based characterization and injection. Cardiomyogenic differentiation of murine MiPs was induced in coculture with neonatal rat cardiomyocytes or via spontaneous differentiation. Neonatal rat cardiomyocytes were harvested from newborn rat pups within 24 hours of birth by digesting the minced hearts in ADS buffer (tissue culture water supplemented with $0.0085 \% \mathrm{NaCl}, 0.0005 \% \mathrm{KCl}, 0.00015 \%$ monohydrated $\mathrm{NaH}_{2} \mathrm{PO}_{4}$, $0.00125 \%$ monohydrated $\mathrm{MgSO}_{4}, 0.00125 \%$ glucose, and $0.00595 \%$ HEPES) (Sigma-Aldrich) supplemented with $0.4 \%$ collagenase II and
$0.6 \%$ pancreatin (Sigma-Aldrich) and subjected to three 20-minute rounds of shaking in a $37^{\circ} \mathrm{C}$-warmed water bath. The neonatal rat cardiomyocytes were further purified using a Percoll gradient $(0.458 \mathrm{~g} / \mathrm{ml}$ to $0.720 \mathrm{~g} / \mathrm{ml}$; GE Healthcare). MiPs were seeded in coculture with rat neonatal cardiomyocytes with a 1:10 $\mathrm{MiP} /$ cardiomyocyte ratio in cardiomyocyte maintenance medium (high-glucose DMEM supplemented with $18 \%$ medium $199,5 \%$ horse serum, $5 \%$ FBS, $1 \%$ penicillin/ streptomycin, and 1\% L-glutamine) (Thermo Fisher Scientific) on gelatin-coated vessels (Nunc, Thermo Fisher Scientific) for 72 hours in $5 \% \mathrm{O}_{2} / 5 \% \mathrm{CO}_{2}$ at $37^{\circ} \mathrm{C}$. Cardiomyogenic spontaneous differentiation was induced by seeding murine MiPs at low density (5,000 cells/ $\mathrm{cm}^{2}$ ) on collagen-coated vessels (Nunc, Thermo Fisher Scientific) in RPMI 20\%/10\% medium (RPMI supplemented with 20\% FBS, 10\% horse serum, $1 \%$ penicillin/streptomycin, and 1\% L-glutamine) for 72 hours in $5 \% \mathrm{O}_{2} / 5 \% \mathrm{CO}_{2}$ at $37^{\circ} \mathrm{C}$, then further matured in DMEM $2 \%$ medium (high-glucose DMEM supplemented with $2 \%$ horse serum, $1 \%$ penicillin/streptomycin, and 1\% L-glutamine) for 24 to 48 hours in $5 \% \mathrm{O}_{2} / 5 \% \mathrm{CO}_{2}$ at $37^{\circ} \mathrm{C}$. Skeletal myogenic differentiation of murine MiPs was induced in coculture with $\mathrm{C} 2 \mathrm{C} 12$ fetal myoblasts or through pulsed overexpression of Pax 3 and Pax7. Murine MiPs were seeded in coculture with $\mathrm{C} 2 \mathrm{C} 12$ fetal myoblasts with a 1:10 MiP/myoblast ratio in RPMI 20\%/10\% medium medium on collagen-coated vessels for 24 hours, then differentiated in DMEM 2\% medium for 96 to 120 hours in $5 \% \mathrm{O}_{2} / 5 \% \mathrm{CO}_{2}$ at $37^{\circ} \mathrm{C}$. Murine MiPs were pulsed with Pax3 and Pax7 through transfection in proliferative conditions with two pSPORT6.1 plasmids bearing Pax3 and Pax7 cDNA sequences and with Lipofectamine (Thermo Fisher Scientific) according to the manufacturer's instructions. The murine MiPs were then differentiated for 10 days in DMEM $2 \%$ medium supplemented with $1 \%$ insulin-transferrinselenium (ITS) (Thermo Fisher Scientific), $100 \mathrm{ng} / \mathrm{ml}$ recombinant noggin (Thermo Fisher Scientific), and $1 \mathrm{mM}$ TGF- $\beta$ inhibitor (Sigma-Aldrich; SB 431542). Transient overexpression of brachyury (pCMV6-Entry; Origene) and Pax7 (as mentioned above) in MiPs was achieved by Lipofectamine-based transfection of small amounts of plasmid, i.e., $100 \mathrm{ng}$ brachyury-bearing and $120 \mathrm{ng}$ Pax7-bearing plasmids per 24-well plate (cells transfected with the empty vectors were used as a control). Then, transfected cells were harvested after 48 hours, analyzed for gene induction, and plated for coculture with myoblasts and for spontaneous differentiation. Osteogenic and adipogenic differentiation of murine MiPs was induced using osteogenic and adipogenic kits (EMD Millipore) according to the manufacturer's instructions. Smooth muscle differentiation was performed on MiPs seeded at low density on collagen-coated vessels in DMEM $2 \%$ supplemented with $50 \mathrm{ng} / \mathrm{ml} \mathrm{TGF-} \beta 1$ (Peprotech). Endothelial differentiation was induced by seeding $10^{5}$ MiPs per well, previously cultured for 2 passages in EBM2 medium (Lonza) on fibronectin-coated plastic, then in EBM2 medium in 48-well plates (Nunc, Thermo Fisher Scientific) coated with a thin layer of Geltrex (Thermo Fisher Scientific). Tubular structures were well formed and apparent 48-72 hours after seeding, and $10 \mu \mathrm{g} / \mu \mathrm{l}$ Dil-Ac-LDL (Thermo Fisher Scientific) was supplemented for 4 hours in culture before proceeding to immunostaining.

Canine and human fibroblasts and MABs were isolated as previously reported (25) and under conditions essentially analogous to those for the murine cells described above. The medium for isolating and growing canine and human MABs consisted of IMDM $10 \%$ supplemented with $10 \% \mathrm{FBS}, 1 \%$ penicillin/streptomycin, $1 \%$ L-glutamine, $1 \%$ sodium pyruvate, $1 \%$ NEAA, $1 \%$ ITS supplement (Thermo Fisher 
Scientific), 0.2\% 2-mercaptoethanol, and $5 \mathrm{ng} / \mathrm{ml}$ bFGF (PeproTech). Reprogramming of canine and human cells was performed under conditions analogous to those for murine cell reprogramming, using retroviral particles carrying human OCT4, SOX2, KLF4, and cMYC and cDNA sequences (pMX vectors; Addgene). Cells were seeded at low confluence on a feeder layer of iMEFs. ESC-like colonies were manually picked 3-4 weeks after transduction, clonally expanded, and characterized for pluripotency marker expression. Canine and human $\mathrm{f}$ - and MAB-iPSCs were cultured on a feeder layer of iMEFs in hiPSC medium (DMEM-F12 supplemented with Ham's F12 Nutrient Mixture [Thermo Fisher Scientific], 20\% knockout serum, 1\% penicillin/ streptomycin, 1\% L-glutamine, 1\% NEAA, 0.2\% 2-mercaptoethanol, and $5 \mathrm{ng} / \mathrm{ml} \mathrm{bFGF}$ [PeproTech]). Differentiation of canine and human $\mathrm{f}$ - and MAB-iPSCs toward MiPs was performed under conditions analogous to those for murine iPSCs, except for the first step of EB formation in hiPSC medium and for a 96-hour duration for each of the three steps. Canine and human MiPs were FACS sorted under conditions analogous to those for murine MiPs, using APC-conjugated antiCD140a, anti-CD140b (Antibodies Online; catalogs ABIN910520 and ABIN457302), and anti-CD44 Abs (eBioscience; catalog 17-0441-81). As freeze/thaw cycles are detrimental to canine cell survival and properties, experiments with canine iPSCs and MiPs were performed with reprogrammed/isolated cells under continuous passaging. Finally, culture of canine and human MiPs and their differentiation toward the different mesodermal lineages (cardiomyogenic, skeletal myogenic, osteogenic, adipogenic, and smooth muscle) were performed following the procedures optimized for murine MiPs.

Molecular assays. Gene expression levels were assayed by SYBR Green qPCR on 1:5 diluted cDNA obtained from $1 \mu \mathrm{g}$ total RNA (SYBR Green mix, SSIII cDNA production kit, and RNA extraction kit; all from Thermo Fisher Scientific) using a ViiA 7384 plate reader (Thermo Fisher Scientific) at a final primer concentration of $100 \mathrm{nM}$; a final volume of 10_l; a $P g k / P G K$ internal reference; and a thermal profile of 15 seconds at $95^{\circ} \mathrm{C}$ and 60 seconds at $60^{\circ} \mathrm{C}, \times 40$ cycles. Primers are listed in the Supplemental material.

Methylation patterns were assayed by means of bisulphite sequencing of $\mathrm{CpG}$ islands located $10 \mathrm{~kb}$ upstream of the transcription start site of the target genes (according to MethPrimer, ref. 36). Genomic DNA (gDNA) was isolated using a Genomic DNA Mini Kit (Thermo Fisher Scientific), then $1 \mu \mathrm{g} / 20 \mu \mathrm{l}$ was bisulphite converted using an EpiTect Bisulphite Kit (QIAGEN). Single CpG island amplicons were amplified by PCR (final primer concentration: $330 \mathrm{nM}$; final volume: $20 \mu \mathrm{l}$; thermal profile: 30 seconds at $95^{\circ} \mathrm{C}, 60$ seconds at $55^{\circ} \mathrm{C}$, and 60 seconds at $72^{\circ} \mathrm{C}, \times 40$ cycles) on a $\mathrm{T} 3000$ Thermocycler (Biometra) using Taq polymerase (Thermo Fisher Scientific), then gel extracted using a Gel Extraction Kit (Thermo Fisher Scientific) and ligated into pGEM plasmids via TA cloning (Promega). Sequencing reactions were prepared with T7 and SP6 primers using BigDye Terminator v3.1 Cycle Sequencing and BigDye Exterminator kits (Thermo Fisher Scientific), sequenced on an Applied Biosystems ABI 3100 Sequencer (performed at the Genomics Core, UZ-KU Leuven, Leuven, Belgium), and analyzed with QUMA on-line software (37). Statistical analysis was performed on average methylation values of 5 sequences per cell clone (3 clones per cell type obtained from independent donors).

Histone mark levels were assayed by ChIP on the same $\mathrm{CpG}$ islands assayed by bisulphite sequencing, adapting previously described conditions (38) to $5 \times 10^{6}$ cell pellets. The DNA polyclonal
Abs $(1 \mu \mathrm{g} / 10 \mu \mathrm{g})$ anti-K9me3 (repressive mark), anti-K4me2 (permissive mark), and anti-K27ac (active mark) (all obtained from Active Motif; catalogs 39142, 39133, and 39765) were used in the ChIP experiments, and protein A-coated sepharose beads (GE Healthcare) were used for the subsequent pulldown. IgG isotype (eBioscience) was used as a negative ChIP control. The initial sonicated gDNA fragment suspension $(5 \mu \mathrm{l}$ of $100 \mu \mathrm{l})$ was used as a reference input. Purification of ChIP and input DNA was performed using a MinElute kit (QIAGEN), and quantification as a percentage of input was performed by SYBR Green qPCR under the conditions described above. Statistical analysis was performed using three qPCR replicates per cell clone (3 clones per cell type obtained from independent donors).

Western blot (WB) analyses were performed on $50 \mu \mathrm{g}$ cell or tissue lysate $(100 \mu \mathrm{g}$ for dystrophin analysis) according to the commonly used procedures in $10 \%$ acrylamide (6\% for dystrophin analysis) hand-cast gels. The following Abs and relative dilutions were used: mouse anti-Sarc $\alpha$ Act (Abcam; catalog ab72592; 1:500); mouse anti-Sgcb (Novocastra, Leica Biosystems; catalog B-SARC-CE; 1:500); mouse anti-dystrophin 3 (interacting with canine and human isoforms; Novocastra, Leica Biosystems; catalog DYS3-CE-S; 1:500); rabbit anti-GAPDH (Sigma-Aldrich; catalog G9545; 1:1,000); and secondary HRP-conjugated Abs (Santa Cruz Biotechnology Inc.; catalogs sc-2005 and sc-2004; 1:500). Bands were detected and imaged with a Bio-Rad Gel Doc using a Pico substrate (Thermo Fisher Scientific) and a Dura substrate for dystrophin analysis. Densitometric analyses were performed on gels loaded, blotted, and detected in parallel with Quantity One software (Bio-Rad).

In vivo injections and functional analyses. Teratoma formation was testing by s.c. injecting $3 \times 10^{5}$ iPSCs at passage 3 into immunodeficient Rag2-null $\gamma c$-null mice (The Jackson Laboratory), using either Matrigel-free murine iPSCs or canine and human iPSCs that were grown in a $1: 1 \mathrm{v} / \mathrm{v}$ matrigel/cell suspension. Teratomas were isolated 4-6 weeks after injection for murine iPSCs and 6-8 weeks after injection for canine and human iPSCs and were further tested for histological, immunostaining, or molecular analyses. Morula aggregation was performed by aggregating trypsin-pretreated $\mathrm{GFP}^{+}$ iPSCs (<10-cell clumps; C57/Bl6 background; passages 3-5) with CD1 morulae (KU Leuven Animal Facility, internal stock mice) freed from the zona pellucida by acidic Tyrode's solution and cultured in KSOM medium (Specialty Media) under preequilibrated mineral oil (Sigma-Aldrich) at $37^{\circ} \mathrm{C}$ in $5 \% \mathrm{CO}_{2}$. The next day, embryos were transferred into the uterus of pseudopregnant Swiss females (KU Leuven Animal Facility, internal stock mice). Chimerism was detected by GFP stereofluorescence, anti-GFP staining of cryosections, and coat chimerism after birth and in the chimeric F1 litter. Dystrophic cardiomyopathic $S g c b$-null mice (23) were divided into randomized groups at 3 months of age $(n=8$, sham; $n=8$, f-MiPs; $n=8$, MAB-MiPs for bulk injections; $n=5$ per cohort for single-cell clone injections) and injected with $\mathrm{GFP}^{+}$murine MiPs (passages 3-7). All mouse groups were kept on cyclosporine-based immunosuppressive treatment throughout the entire experiment. MiPs were exposed to RPMI $20 \% / 10 \%$ medium for 48 hours, then injected in parallel into the left ventricular myocardium and into both femoral arteries of each animal under isofluorane anesthesia $\left(5 \times 10^{5}\right.$ cells $/ 5 \times 2.5 \mu \mathrm{l}$ in the myocardium; $5 \times 10^{5}$ cells $/ 100 \mu \mathrm{l}$ per femoral artery). Sham controls received equal treatment and amounts of cell-free saline solution. Engraftment, regeneration, and functional outcomes were 
investigated 4 and 8 weeks after injection. Muscle force and offtarget engraftment analyses were conducted 8 weeks after injection. Immunodeficient Rag2-null $\gamma \mathrm{c}$-null mice (The Jackson Laboratory) were divided into randomized groups at 2 months of age $(n=7$, sham; $n=7$, f-MiPs; $n=7$, MAB-MiPs) and injected with canine MiPs (passages $3-7)$. Ten days before cardiac injection, the hind limb skeletal muscles of mice from all groups were locally injected with cardiotoxin ( $50 \mu \mathrm{l} /$ muscle, $10 \mu \mathrm{M}$ solution). Seven days before cardiac injection, mice were injected with cells or sham into both femoral arteries $\left(5 \times 10^{5}\right.$ cells $/ 100 \mu$ l per femoral artery) under isofluorane anesthesia. Cardiac injection was performed directly into the left ventricular myocardium $\left(5 \times 10^{5}\right.$ cells $\left./ 10 \mu \mathrm{l}\right) 1$ hour after coronary artery ligation. Engraftment, regeneration, and functional outcomes were investigated 4 weeks after injection. Immunodeficient, dystrophic $S g c b$-null Rag2-null $\gamma$ c-null mice were generated by crossing the aforementioned dystrophic and immunodeficient mice strains until achieving homozygosity in all mutated alleles, as determined by PCR of gDNA using dedicated JAX primers (The Jackson Laboratory). Sgcbnull Rag2-null $\gamma c$-null mice were injected and analyzed according to the procedures used for dystrophic mice. High-resolution digital ultrasound images were obtained by an experienced echocardiographer using the Vevo 2100 Imaging System (VisualSonics) with a $30-\mathrm{MHz}$ probe. Mice were anesthetized using $1 \%$ isofluorane in oxygen, positioned on the system's heating pad in order to maintain normothermia, and continuously monitored. Prewarmed ultrasound gel was applied on the shaved thorax. B-mode-based 3D reconstruction was performed using the VisualSonics rail system with a fixed probe, with ECG and respiratory gating. Fractional shortening (FS) was calculated on the basis of left ventricular internal dimension in diastole and systole (LVIDd/s) values, whereas end-diastolic volume (EDV), cardiac output (CO), and stroke volume (SV) were calculated on the basis of 3D analysis. Raw data were collected in a blinded fashion. Treadmill testing was conducted on $10^{\circ}$ incline treadmill belt with 1 $\mathrm{m} / \mathrm{min}^{2}$ acceleration at a starting speed of $10 \mathrm{~m} / \mathrm{min}$. The mouse run was stopped after 5 or more consecutive seconds on the pulsed grill. Muscle force assessment was performed on freshly isolated EDL muscles upon sacrifice, using a 1200A in vitro muscle test system (Aurora Scientific). Muscle force was probed upon 20 iterated bouts of isometric contractions $(200-\mathrm{Hz}, 80-\mathrm{V}, 0.5-$ millisecond stimulation, 0.5 -second tetanus at 10 -second intervals in $30^{\circ} \mathrm{C}$ ) in dedicated buffer $\left(1.2 \mathrm{mM} \mathrm{KH}_{2} \mathrm{PO}_{4}, 0.57 \mathrm{mM} \mathrm{MgSO}_{4}{ }^{*} 7 \mathrm{H}_{2} \mathrm{O}, 2 \mathrm{mM} \mathrm{CaCl}_{2}{ }^{*} 2 \mathrm{H}_{2} \mathrm{O}\right.$, $10 \mathrm{mM}$ HEPES, $0.5 \mathrm{mM} \mathrm{MgCl}_{2}^{*} 6 \mathrm{H}_{2} \mathrm{O}, 0.5 \mathrm{mM} \mathrm{MgCl}_{2}{ }^{*} 6 \mathrm{H}_{2} \mathrm{O}, 4.5 \mathrm{mM}$ $\mathrm{KCl}, 120 \mathrm{mM} \mathrm{NaCl}, 0.7 \mathrm{mM} \mathrm{Na} \mathrm{HPO}_{4}, 1.5 \mathrm{mM} \mathrm{NaH} \mathrm{PO}_{4}, 10 \mathrm{mM}$ D-glucose, and $15 \mathrm{mM} \mathrm{NaHCO}_{3}$, pH 7.3; Sigma-Aldrich). Data were analyzed as the percentage of maximum absolute force of the input sham muscles. CK levels were measured under resting conditions ( $>24$ hours after the last treadmill test) in serum obtained from more than $50 \mu$ blood (withdrawn from the tail vein). CK level quantitation was performed using the Creatine Kinase Activity Colorimetric Assay kit (BioVision) according to the manufacturer's instructions for sample preparation and standard curve assessment.

Stereomicroscopic and immunofluorescence analyses. Whole fluorescence imaging of injected/chimeric tissues and embryos was performed on an Olympus SZX12 stereomicroscope with analySIS getIT software (2-second exposure for GFP, 0.2-second exposure for bright-field, semirefringent bottom) for chimeric embryos and tissues and murine MiP in vivo experiments and with a Zeiss SteREO
Discovery V12 microscope with Axio Imaging software (2-second exposure for GFP, 0.2-second exposure for bright-field, semirefringent bottom). Immunofluorescence staining was performed following the commonly used Triton-based (Sigma-Aldrich) permeabilization steps, donkey serum (Sigma-Aldrich) background blocking, overnight incubation with primary $\mathrm{Ab}$ at $4^{\circ} \mathrm{C}$, a 1-hour incubation with 1:500 Alexa Fluor-conjugated donkey secondary Abs (Thermo Fisher Scientific), and a final counterstaining with Hoechst. The primary Abs and relative dilutions used were as follows: rabbit anti-nanog (Abcam; catalog ab80892; 1:500); mouse anti-TRA-1-60 (Santa Cruz Biotechnology Inc.; catalog sc-21705; 1:100); rabbit anti-laminin (Sigma-Aldrich; catalog L9393; 1:300); goat anti-GFP (Abcam; catalog ab5450; 1:500); mouse antimyosin heavy chain (anti-MyHC) (Developmental Studies Hybridoma Bank [DSHB]; MF20; 1:3); mouse anti-Sarc $\alpha$ Act (Abcam; catalog ab72592; 1:300); rabbit anti-Myocd (Santa Cruz Biotechnology Inc.; catalog sc-33766; 1:100); mouse anti-Sgcb (Novocastra, Leica Biosystems; catalog B-SARC-CE; 1:100); mouse anti-calponin (Dako; catalog M3556; 1:300); canine- and human-specific mouse antidystrophin 3 (Novocastra, Leica Biosystems; catalog DYS3-CE-S; 1:100); mouse anti-Pax3 (R\&D Systems; catalog MAB2457; 1:300); mouse anti-AP (R\&D Systems; catalog FAB1448P; 1:500); mouse anti-Pax7 (DSHB; catalog PAX7; 1:10); mouse anti-slow MyHC (Sigma-Aldrich; catalog M8421; 1:400); mouse anti-fast MyHC (Sigma-Aldrich; catalog M4276; 1:400); rabbit anti-human Sarc $\alpha$ Act (Abcam; catalog ab62298; 1:300); and mouse anti- $\alpha$-smooth muscle actin (anti- $\alpha$-SMA) (Sigma-Aldrich; catalog A2547; 1:300). Imaging was performed on an ECLIPSE Ti microscope with Image-Pro Plus 6.0 software (both from Nikon). Quantitation of engraftment and satellite cells and fibers was performed with ImageJ software (NIH) on at least 10 fields across the left ventricle and gastrocnemius muscle.

TALEN-mediated genome editing. GRMD mutation $(\mathrm{A} \rightarrow \mathrm{G}$ transition, ref. 39) was checked by gDNA sequencing in our WT and GRMD canine cell samples. TALEN vectors were designed to recognize target regions at $+33 \mathrm{nt}$ and $+66 \mathrm{nt}$ downstream of the mutation site, and a donor matrix was designed to carry the therapeutic $\mathrm{G} \rightarrow \mathrm{A}$ transition at $+0 \mathrm{nt}$ and a silent mutation at $+63 \mathrm{nt}$ from the mutation site $(A \rightarrow G)$ in order to introduce a single SphI restriction site (Cellectis). TALEN vectors $(1 \mu \mathrm{g} / \mathrm{plasmid})$, donor matrix $(3 \mu \mathrm{g})$, and a GFP-carrying plasmid $(1 \mu \mathrm{g})$ were nucleofected into canine GRMD iPSCs $\left(10^{6} \mathrm{cells} / 800\right.$ $\mu \mathrm{l}$ phenol red-free RPMI medium; Thermo Fisher Scientific) using the Gene Pulser Xcell (BioRad). Putative single-cell, nucleofected, transiently $\mathrm{GFP}^{+}$clones were selected, expanded, recloned by singlecell dilution, and checked by SphI assay and by sequencing. An SphI assay was performed on $0.5 \mu$ g PCR-amplified, gel-extracted gDNA amplicons (primers: forward, TGAAAAGTGTTATTGAGGGGTAA; reverse, TCCAGAAATGTACCGACCTT; thermal profile: 30 seconds at $95^{\circ} \mathrm{C}, 60$ seconds at $57^{\circ} \mathrm{C}$, and 60 seconds at $72^{\circ} \mathrm{C}, \times 40$ cycles) by overnight incubation at $37^{\circ} \mathrm{C}$ with $5 \mathrm{U} \mathrm{SphI}$ isoschizomer (PaeI; Thermo Fisher Scientific) in a $30-\mu$ final volume reaction. Sequencing was performed on TA-cloned amplicons under the conditions described above (40 sequences/clone).

Statistics. The sample size for in vitro and in vivo experiments was calculated using a Sample Size Calculator (http://www.stat.ubc. ca/ rollin/stats/ssize/index.html; parameters: power.80; alpha.05). When applicable, sample size analysis was based on average values obtained from preliminary optimization and validation trials. For 
statistical comparisons involving chimeric mice, a Mann-Whitney $U$ test was applied and significance scored when the $P$ value was less than 0.05. For in vivo analyses, a Kruskal-Wallis test followed by Mann-Whitney $U$ test comparing 2 target populations were performed, and significance was scored when $P$ was less than 0.05 for both tests. For gene expression analyses, 2-way ANOVA was applied to patterns measured over time (significance was scored when $P$ was less than 0.05 for both interaction [cells] and column [stages] parameters), and an unpaired homoscedastic $t$ test was applied to fversus MAB-MiP comparisons (a $P$ value of less than 0.05 was considered significant). For methylation pattern analyses, 1-way ANOVA with Bonferroni's multiple comparisons test was applied to methylation values expressed as a percentage of methylated $\mathrm{CpG}$ s versus total $\mathrm{CpGs}$, and significance was achieved when $P$ was less than 0.05. For histone mark analyses, 2-way ANOVA with Bonferroni's multiple comparisons test was applied to histone enrichment values expressed as a percentage of input, and significance was achieved for Q1 and Q2 when $P$ was less than 0.05 for at least 2 of 3 histone mark data pools. All statistical analyses were conducted using GraphPad Prism 5.0 (GraphPad Software).

Study approval. All protocols and experiments involving mice and murine sampling were performed in compliance with Belgian law and with approval of the ethics committee of KU Leuven. Collection of canine samples was performed in compliance with French law and with approval of the ethics committee of the Maison Alford Veterinary School. Human fetal tissues were obtained from patients who required an induced abortion during the first trimester at IRCCS University Hospital San Matteo Foundation, where the surgical termination of pregnancy was also performed. The patients included in the study provided written informed consent before the tissue collection, and the use of patients' samples was approved by the Human Research Ethics Committee of the IRCCS University Hospital San Matteo Foundation.

\section{Author contributions}

MQ designed and performed experiments, analyzed data, provided funding, and drafted the manuscript. M Swinnen, GG, JC, IB, GC, EC, HG, LT, and MM performed experiments and analyzed data. GP, CMV, SB, SJ, and M Sampaolesi analyzed experiments, drafted the manuscript, and provided funding.

\section{Acknowledgments}

This work was funded by the "Opening The Future" Campaign (EJJ-OPTFUT-02010); the Cardio Repair European Multidisciplinary Initiative (CARE-MI FP7); the Association Française contre les Myopathies (AFM); the Cassa di Risparmio delle Provincie Lombarde (CARIPLO); the Fonds voor Wetenschappelijk Onderzoek (FWO) (grants G060612N, G0A8813N, and G088715N); the Geconcerteerde Onderzoeksacties (GOA) (grant 11-012); the Interuniversity Poles of Attraction (IUAP) (grant VII/07); and the Onderzoek Traject (OT) (grant 09-053). M. Quattrocelli is supported by the FWO (postdoctoral fellowship 1263314N and travel grant V448715N) and the AFM (Trampoline grant 18373). We are grateful to C.L. Mummery (Leiden University Medical Center, Leiden, The Netherlands) for suggestions on the manuscript and to M. Chuah and T. Vandendriessche (VUB, Brussels, Belgium) for the GFP transposon. We thank Christina Vochten and Vicky Raets for their professional administrative assistance. We would also like to thank Paolo Luban and Rondoufonds voor Duchenne Onderzoek for their kind donations.

Address correspondence to: Maurilio Sampaolesi, Translational Cardiomyology Lab, Department of Development and Regeneration, KU Leuven, Herestraat 49 - O\&N4 - bus 814, 3000 Leuven, Belgium. Phone: 32.16.330295; E-mail: maurilio.sampaolesi@ med.kuleuven.be.
1. Doulatov S, Daley GQ. Development. A stem cell perspective on cellular engineering. Science. 2013;342(6159):700-702.

2. Costamagna D, et al. Fate choice of postnatal mesoderm progenitors: skeletal versus cardiac muscle plasticity. Cell Mol Life Sci. 2014;71(4):615-627.

3. Emery AE. The muscular dystrophies. Lancet. 2002;359(9307):687-695.

4. Verhaert D, Richards K, Rafael-Fortney JA, Raman SV. Cardiac involvement in patients with muscular dystrophies: magnetic resonance imaging phenotype and genotypic considerations. Circ Cardiovasc Imaging. 2011;4(1):67-76.

5. McNally EM, et al. Contemporary cardiac issues in duchenne muscular dystrophy. Circulation. 2015;131(18):1590-1598.

6. Cao N, et al. Highly efficient induction and long-term maintenance of multipotent cardiovascular progenitors from human pluripotent stem cells under defined conditions. Cell Res. 2013;23(9):1119-1132.

7. Darabi R, et al. Human ES- and iPS-derived myogenic progenitors restore DYSTROPHIN and improve contractility upon transplantation in dystrophic mice. Cell Stem Cell. 2012;10(5):610-619.

8. Polo JM, et al. Cell type of origin influences the molecular and functional properties of mouse induced pluripotent stem cells. Nat Biotechnol. 2010;28(8):848-855.

9. $\mathrm{Kim} \mathrm{K}$, et al. Epigenetic memory in induced pluripotent stem cells. Nature. 2010;467(7313):285-290.

10. Sanchez-Freire V, et al. Effect of human donor cell source on differentiation and function of cardiac induced pluripotent stem cells. J Am Coll Cardiol. 2014;64(5):436-448.

11. Quattrocelli M, et al. Intrinsic cell memory reinforces myogenic commitment of pericyte-derived iPSCs. J Pathol. 2011;223(5):593-603.

12. Magli A, et al. Pax 3 and Tbx 5 specify whether PDGFR $\alpha+$ cells assume skeletal or cardiac muscle fate in differentiating ES cells. Stem Cells. 2014;32(8):2072-2083.

13. Chan SS, et al. Mesp1 patterns mesoderm into cardiac, hematopoietic, or skeletal myogenic progenitors in a context-dependent manner. Cell Stem Cell. 2013;12(5):587-601.

14. Ivey KN, et al. MicroRNA regulation of cell lineages in mouse and human embryonic stem cells. Cell Stem Cell. 2008;2(3):219-29.

15. Kim K, et al. Donor cell type can influence the epigenome and differentiation potential of human induced pluripotent stem cells. Nat Biotechnol. 2011;29(12):1117-1119.

16. Meng $\mathrm{H}$, et al. DNA methylation, its medi- ators and genome integrity. Int J Biol Sci. 2015;11(5):604-617.

17. Sdek P, Oyama K, Angelis E, Chan SS, Schenke-Layland K, MacLellan WR. Epigenetic regulation of myogenic gene expression by heterochromatin protein $1 \alpha$. PLoS One. 2013;8(3):e58319.

18. Yu XY, et al. High levels of glucose induce "metabolic memory" in cardiomyocyte via epigenetic histone $\mathrm{H} 3$ lysine 9 methylation. $\mathrm{Mol}$ Biol Rep. 2012;39(9):8891-8898.

19. Jiang L, et al. ZBED6 modulates the transcription of myogenic genes in mouse myoblast cells. PLoS One. 2014;9(4):e94187.

20. Schlesinger J, et al. The cardiac transcription network modulated by Gata4, Mef2a, Nkx2.5, Srf, histone modifications, and microRNAs. PLoS Genet. 2011;7(2):e1001313.

21. Balog J, et al. Correlation analysis of clinical parameters with epigenetic modifications in the DUX4 promoter in FSHD. Epigenetics. 2012;7(6):579-84.

22. Zhang Z, Zhang MQ. Histone modification profiles are predictive for tissue/cell-type specific expression of both protein-coding and microRNA genes. BMC Bioinformatics. 2011;12:155.

23. Durbeej M, et al. Disruption of the $\beta$-sarcoglycan gene reveals pathogenetic complexity of 
limb-girdle muscular dystrophy type 2E. Mol Cell. 2000;5(1):141-151.

24. Sampaolesi M, et al. Mesoangioblast stem cells ameliorate muscle function in dystrophic dogs. Nature. 2006;444(7119):574-579.

25. Quattrocelli M, Palazzolo G, Perini I, Crippa S, Cassano M, Sampaolesi M. Mouse and human mesoangioblasts: isolation and characterization from adult skeletal muscles. Methods Mol Biol. 2012;798:65-76.

26. Goldman JP, Blundell MP, Lopes L, Kinnon C, Di Santo JP, Thrasher AJ. Enhanced human cell engraftment in mice deficient in RAG2 and the common cytokine receptor $\gamma$ chain. Br J Haematol. 1998;103(2):335-342.

27. Wood AJ, et al. Targeted genome editing across species using ZFNs and TALENs. Science. 2011;333(6040):307.

28. Townsend D, Yasuda S, Li S, Chamberlain JS, Metzger JM. Emergent dilated cardiomyopathy caused by targeted repair of dystrophic skeletal muscle. Mol Ther. 2008;16(5):832-835.

29. Laurent LC, et al. Dynamic changes in the copy number of pluripotency and cell proliferation genes in human ESCs and iPSCs during reprogramming and time in culture. Cell Stem Cell. 2011;8(1):106-118.

30. Dellavalle A, et al. Pericytes of human skeletal muscle are myogenic precursors distinct from satellite cells. Nat Cell Biol. 2007;9(3):255-267.

31. Dellavalle A, et al. Pericytes resident in postnatal skeletal muscle differentiate into muscle fibres and generate satellite cells. Nat Commun. 2011;2:499.

32. Messina G, et al. Skeletal muscle differentiation of embryonic mesoangioblasts requires pax3 activity. Stem Cells. 2009;27(1):157-64.

33. Fuoco $\mathrm{C}$, et al. In vivo generation of a mature and functional artificial skeletal muscle. $Е M B O M O l$ Med. 2015;7(4):411-422.

34. Tan KY, Eminli S, Hettmer S, Hochedlinger
$\mathrm{K}$, Wagers AJ. Efficient generation of iPS cells from skeletal muscle stem cells. PLoS One. 2011;6(10):e26406.

35. Kim J, et al. An iPSC line from human pancreatic ductal adenocarcinoma undergoes early to invasive stages of pancreatic cancer progression. Cell Rep. 2013;3(6):2088-2099.

36. Li LC, Dahiya R. MethPrimer: designing primers for methylation PCRs. Bioinformatics. 2002;18(11):1427-1431.

37. Kumaki Y, Oda M, Okano M. QUMA: quantification tool for methylation analysis. Nucleic Acids Res. 2008;36(Web Server issue):W170-W175.

38. Carey MF, Peterson CL, Smale ST. Chromatin immunoprecipitation (ChIP). Cold Spring Harb Protoc. 2009;2009(9):pdb.prot5279.

39. Sharp NJ, et al. An error in dystrophin mRNA processing in golden retriever muscular dystrophy, an animal homologue of Duchenne muscular dystrophy. Genomics. 1992;13(1):115-121. 